KCL-TH-04-16

MIFP-04-28/04

hep-th/0412245

October 28, 2018

\title{
The supermembrane revisited
}

\author{
P.S. Howe ${ }^{1}$ and E. Sezgin ${ }^{2}$ \\ 1 Department of Mathematics, King's College, London, UK \\ 2 Center for Theoretical Physics, Texas A\&M University, College Station, TX 77843, USA
}

\begin{abstract}
The M2-brane is studied from the perspective of superembeddings. We review the derivation of the M2-brane dynamics and the supergravity constraints from the standard superembedding constraint and we discuss explicitly the induced $d=3, N=8$ superconformal geometry on the worldvolume. We show that the gauged supermembrane, for a target space with a $U(1)$ isometry, is the standard D2-brane in a type IIA supergravity background. In particular, the D2-brane action, complete with the Dirac-Born-Infeld term, arises from the gauged Wess-Zumino worldvolume 4-form via the brane action principle. The discussion is extended to the massive D2-brane considered as a gauged supermembrane in a massive $D=11$ superspace background. Type IIA supergeometry is derived using Kaluza-Klein techniques in superspace.
\end{abstract}




\section{Contents}

1 Introduction $\quad 2$

2 Superembeddings: connection-free formalism $\quad 3$

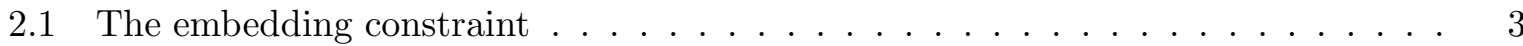

2.2 Linearised embeddings . . . . . . . . . . . . . . . . . . . 6

3 Lorentzian supergeometry and superembeddings $r$

3.1 Target superspace geometry . . . . . . . . . . . . . . . . . . 8

3.2 Superembeddings . . . . . . . . . . . . . . . . . . . . . . 9

4 The dynamics of the M2-brane $\quad 14$

4.1 The torsion equations . . . . . . . . . . . . . . . . . . 14

4.2 The equations of motion in component form . . . . . . . . . . . . . . . 16

4.3 Kappa symmetry . . . . . . . . . . . . . . . . . . . . . . . . 20

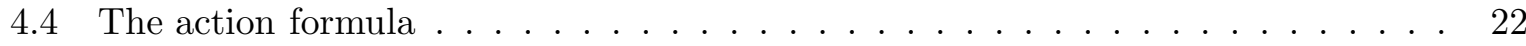

5 The induced supergeometry $\quad 23$

5.1 The $d=3, N=8$ conformal supergravity multiplet . . . . . . . . . . . 23

5.2 Super-Weyl transformations . . . . . . . . . . . . . . . . . . . . . 25

6 The gauged M2-brane $\quad 26$

6.1 Gauged M2-brane superembedding . . . . . . . . . . . . . . . . . . 27

6.2 Geometrical structure, Wess-Zumino form and action . . . . . . . . . . . . . . . 29

7 Massive branes $\quad 32$

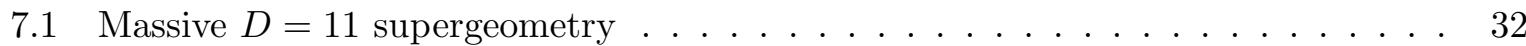

7.2 The massive gauged superembedding . . . . . . . . . . . . . . . . . 34

7.3 The Wess-Zumino form . . . . . . . . . . . . . . . . . . 35

8 Conclusions $\quad 36$

$\begin{array}{ll}\text { A Conventions } & 36\end{array}$

$\begin{array}{ll}\text { B Riemannian embeddings } & 39\end{array}$

C Kaluza-Klein reduction of $D=11$ superspace $\quad 42$ 


\section{Introduction}

The supermembrane [1] is an important ingredient of M-theory. It arises as a solution of elevendimensional supergravity [2], the low-energy limit of M-theory, and is one of the candidates for an underlying microscopic definition of the theory, at least in a particular background via the matrix model $[3,4]$. The type IIA string arises from the supermembrane via double dimensional reduction [5], while vertical reduction leads to the effective action for the D2-brane [6, 7].

A membrane traces out a 3-dimensional worldvolume as it moves through spacetime, but the supermembrane also has fermionic degrees of freedom and can be viewed as a bosonic extended object moving through superspace. This gives rise to a Green-Schwarz type action which is a spacetime supersymmetric version of the Nambu-Goto action together with a Wess-Zumino term. The ratio between the two terms in the action is fixed, as in the Green-Schwarz superstring [8], by the requirement that the action be invariant under a local fermionic symmetry [1] called kappa-symmetry [9]. This symmetry implies that the fully gauge-fixed worldvolume theory is supersymmetric. It was realised some time ago, first in the case of superparticles $[10,11]$ moving in low spacetime dimensions and subsequently for other extended objects, reviewed in [12], that kappa-symmetry can be re-interpreted as local worldvolume supersymmetry. This is particularly clear from the point of view of superembeddings where the local supersymmetry on the bosonic worldvolume can be identified with the leading component (in a $\theta$ expansion) of an odd superworldvolume diffeomorphism. The supermembrane itself was discussed from this point of view in [13] (see [12] for a review). The superembedding formalism can be applied to all BPS branes, including D-branes [14]; in particular, it was used to obtain the dynamics of the M5-brane in a covariant formalism $[15,16]$. The dynamics of the M5-brane were subsequently derived in a suitably modified Green-Schwarz formalism $[17,18]$.

There is a universal constraint which is imposed in the superembedding formalism which, in many cases, determines the worldvolume dynamics, the constraints on the target space supergeometry and the induced geometry on the worldvolume [14, 19]. This is true for M-branes and most D-branes; all D-branes can be accommodated in the formalism if open strings ending on them are taken into account [20]. This approach leads naturally to the equations of motion, but an action principle has also been developed in the superembedding formalism [21, 22]. Moreover, the basic embedding constraint is closely related to the characteristic form of kappa-symmetry transformations. The superembedding formalism also provides a convenient framework to study higher-derivative corrections to the target space supergeometry or to the worldvolume dynamics. This requires the embedding constraint to be modified as was shown in the case of the M2-brane in [23], and more recently for the M5-brane in [24]. Higher-derivative corrections for the D9-brane, where the basic embedding condition does not suffice to give the lowest-order dynamics, have also been constructed in the superembedding formalism [25].

In this paper we focus on the lowest order dynamics of the M2-brane in the superembedding framework. We begin by reviewing the superembedding formalism from two points of view, with and without introducing a connection on the target superspace. We show how the embedding constraint requires the constraints on the target space supergeometry to be those that describe on-shell $D=11$ supergravity. We then provide the full details of the derivation of the M2brane dynamics, including the equations of motion in a supergravity background in component form. We review the brane action principle and use it to derive the Green-Schwarz action for the supermembrane starting from a closed 4 -form on the worldvolume. We show how one can determine the worldvolume supervielbein and give expressions for the bosonic worldvolume 
dreibein and the worldvolume gravitino as composite fields. We discuss the structure of the induced $d=3, N=8$ supergeometry and show that it coincides with that of off-shell $d=3, N=$ 8 conformal supergravity.

In section 6 we consider the gauged supermembrane. This has been discussed in components in $[26,27]$, and in superspace in [28], but has not been discussed previously in the superembedding formalism. It is shown that the gauged superembedding formalism leads directly to the D2-brane in IIA superspace without the need to dualise the additional scalar coming from the eleventh direction. This is done at the level of the equations of motion via the natural gauged version of the embedding constraint; it is also shown how the D2-brane action can be derived using an appropriate modification of the construction of the Wess-Zumino term in gauged sigma models. In section 7 we study the massive D2-brane [29] starting from $D=11[26,27]$. The $D=11$ supergeometry corresponding to the lifted massive type IIA supergravity is described. This is not truly eleven-dimensional since it relies on the presence of a Killing vector, but it facilitates the construction of the brane theory via gauged superembedding. As in the massless case one finds that the gauged superembedding formalism leads directly to the equations of motion of the D2-brane. The action can also be constructed. As expected, it includes a Chern-Simons term for the Born-Infeld gauge field proportional to the mass.

Our conventions are summarised in Appendix A. In Appendix B we give an of the basic features of embeddings in Riemannian geometry, and in Appendix $\mathrm{C}$ we obtain the superspace constraints of type IIA supergravity by Kaluza-Klein reduction from eleven-dimensional superspace.

\section{Superembeddings: connection-free formalism}

\subsection{The embedding constraint}

In ordinary Lorentzian geometry the basic object is the metric tensor $g$ which can be brought to the standard form $\eta_{a b}$ in an orthonormal basis. If $M$ is an embedded submanifold of $\underline{M}$, the metric $g$ on $\underline{M}$ induces a metric $g$ on $M$ in the standard way. However, in supergeometry, the basic object is not $\eta_{a b}$ but rather the Dirac matrices $\left(\Gamma^{c}\right)_{\alpha \beta}$. This gives rise to a threeindex object but it is not a tensor on the whole space. In order to facilitate the discussion of superembeddings it is therefore useful to formalise this structure. We shall do it for the case of $D=11$ but the discussion can be generalised to arbitrary superspaces. However, not only is $D=11$ the case of interest for us it is also particularly simple as we shall see.

Let $\underline{M}$ be an (11|32)-dimensional (real) supermanifold. We suppose that we are given a choice of odd tangent bundle $\underline{F} \subset \underline{T}$, where $\underline{T}$ is the tangent bundle. We can define a three-index tensor, which we call the Frobenius tensor, as follows: let $\left(E_{\underline{\alpha}}\right)$ and $\left(E^{\underline{a}}\right)$ be bases for $\underline{F}$ and $\underline{B}^{*}$, the even cotangent bundle, then the components of the Frobenius tensor in such a basis, denoted by $T_{\underline{\alpha} \underline{c}}$, are given by

$$
T_{\underline{\alpha \beta}} \underline{c}=-\left\langle\left[E_{\underline{\alpha}}, E_{\underline{\beta}}\right], E^{\underline{c}}\right\rangle,
$$

where $\langle$,$\rangle denotes the usual pairing between vectors and forms. This tensor is usually called$ the dimension zero torsion tensor but it does not require the introduction of a connection to be well-defined. The motivation for the nomenclature is that the vanishing of this tensor would imply that $\underline{F}$ is involutive so that the Frobenius theorem would apply, i.e. there would be purely 
odd integral submanifolds of $\underline{M}$ whose tangent spaces coincide with $\underline{F}$. In the present context, this tensor is actually maximally non-integrable in the sense that the even tangent bundle is generated from the odd tangent bundle via the obvious map $\wedge^{2} \underline{F} \rightarrow \underline{B}$ defined by the Frobenius tensor. From the definition of the latter, this is related to taking commutators of odd vector fields and so can be thought of as a statement of supersymmetry.

If we assume that the above structure is irreducible, i.e. it does not break up into the direct sum of two lower-dimensional ones, then there exists a choice of basis such that the Frobenius tensor can be brought to the form

$$
T_{\underline{\alpha \beta}}^{\underline{c}}=-i\left(\left(\Gamma^{\underline{c}}\right)_{\underline{\alpha \beta}}+\left(\Gamma^{\underline{b c}}\right)_{\underline{\alpha \beta}} X_{\underline{b c}}^{\underline{a}}+\left(\Gamma^{\underline{b c d e f}}\right)_{\underline{\alpha \beta}} Y_{\underline{b c d e f}} \underline{a}\right) .
$$

Here $X$ and $Y$ are both traceless and their totally antisymmetric parts vanish when the $\underline{a}$ index is lowered using the standard Lorentzian metric $\eta_{\underline{a b}}$. Note that this is a scale invariant statement since it remains true for any metric in the same conformal class as $\eta$. This is the most general form of the Frobenius tensor possible in the sense that it can be achieved by using all the freedom one has to choose the bases $\left(E_{\underline{\alpha}}\right),\left(E^{\underline{a}}\right)$ leaving only $C \operatorname{Spin}(1,10):=\operatorname{Spin}(1,10) \times \mathbb{R}^{*}$ transformations. ${ }^{1}$ The supergravity equations of motion are implied, at least locally, when the Frobenius tensor takes the simple form

$$
T_{\underline{\alpha \beta}}^{\underline{c}}=-i\left(\Gamma^{\underline{c}}\right)_{\underline{\alpha \beta}} .
$$

This is not immediately obvious but can be proven most simply by working systematically through the Bianchi identities using the connection formalism [30]. One can restate this by saying that the equations of motion of supergravity will follow if the Frobenius tensor is invariant under $C \operatorname{Spin}(1,10$. In practice, the tensors $X$ and $Y$ are non-zero only when one includes higher derivative corrections to supergravity. In this case they will be functions of the supergravity fields involving a dimensionful parameter so that local scale invariance will be explicitly broken.

We now consider the embedding of the worldvolume $M$ in $\underline{M}$. For the 2-brane $M$ is a (3|16)dimensional supermanifold. The natural superembedding condition, which holds for all branes at least at leading order in derivatives, states that there is a choice of odd tangent bundle $F$ for $M$ such that $F \subset \underline{F}$ at all points of $M$. Dually one has $B^{*} \subset \underline{B}$. The only other requirement we need to impose on the embedding is that the metric induced on $B^{*}$ (from $\eta_{a b}$ ) should be Lorentzian. Again this condition is automatically conformally invariant.

The embedding condition induces a Frobenius tensor on $M$ in a straightforward manner. We introduce local bases $\left(E_{\alpha}\right),\left(E^{a}\right)$ for $F$ and $B^{*}$ respectively and note that $F \subset \underline{F}$ implies that

$$
E_{\alpha}=E_{\alpha} \underline{\alpha} E_{\underline{\alpha}}
$$

for some $16 \times 32$ matrix $E_{\alpha} \underline{\underline{\alpha}}$. Equivalently,

$$
E_{\alpha} \underline{a}=0
$$

Dually one has

\footnotetext{
${ }^{1}$ In fact, one could have other signatures of spacetime, but we shall not consider such cases here.
} 


$$
E^{\underline{a}}=E^{a} E_{a}^{\underline{a}}
$$

where on the left-hand side $E^{\underline{a}}$ is pulled-back onto $M$. One then has

$$
\begin{aligned}
\left\langle\left[E_{\alpha}, E_{\beta}\right], E^{\underline{a}}\right\rangle & =E_{\alpha} \underline{\alpha} E_{\beta} \underline{\beta}\left\langle\left[E_{\underline{\alpha}}, E_{\underline{\beta}}\right], E^{\underline{a}}\right\rangle \\
& =\left\langle\left[E_{\alpha}, E_{\beta}\right], E^{a}\right\rangle E_{a} \underline{a}
\end{aligned}
$$

at any point $p \in M$. Thus the Frobenius tensors are related by

$$
E_{\alpha} \underline{\underline{\alpha}} E_{\beta} \underline{\underline{\beta}} T_{\underline{\alpha} \beta^{\underline{c}}}=T_{\alpha \beta}^{c} E_{c^{\underline{c}}}^{\underline{c}} .
$$

If $\left(E_{\underline{\alpha}}\right)$ is a spin basis for $\underline{F}$ any other such basis will be related to it by an element $u$ of $\operatorname{Spin}(1,10)$ up to a conformal factor which we shall ignore for the moment. We write $u=\left(u_{\alpha} \underline{\underline{\alpha}}, u_{\alpha^{\prime}} \underline{\alpha}\right)$, with $\alpha^{\prime}=1, \ldots 16$. Since $E_{\alpha} \underline{\alpha}$ has maximal rank there will be a choice of $u$ such that $E_{\alpha}$ is related to $u_{\alpha} \underline{\alpha} E_{\underline{\alpha}}$ by a non-singular matrix. Hence, without loss of generality, we can write

$$
E_{\alpha} \underline{\alpha}=A_{\alpha}^{\beta} u_{\beta^{\underline{\alpha}}}+B_{\alpha}^{\beta^{\prime}} u_{\beta^{\prime}} \underline{\alpha}
$$

where $\operatorname{det} A \neq 0$. Making a change of basis for $F$ we arrive at

$$
E_{\alpha} \underline{\alpha}=u_{\alpha} \underline{\alpha}+h_{\alpha}^{\beta^{\prime}} u_{\beta^{\prime}} \underline{\alpha} .
$$

On the bosonic space $B^{*}$ the situation resembles more closely the case of a Lorentzian embedding and we may choose, again up to a conformal factor

$$
E_{a} \underline{a}=u_{a}^{\underline{a}}
$$

where $\left(u_{a^{\underline{a}}}, u_{a^{\prime}} \underline{\underline{a}}\right)$ is the element of $S O(1,10)$ corresponding to $u=\left(u_{\alpha} \underline{\underline{\alpha}}, u_{\alpha^{\prime}} \underline{\underline{\alpha}}\right) \in \operatorname{Spin}(1,10)$. Thus, at any point $p \in M$, the embedding is specified by $u_{a} \underline{a}, u_{\alpha} \underline{\underline{\alpha}}$ and $h_{\alpha}{ }^{\beta^{\prime}}$.

We can now decompose equation (2.8) into components tangent and normal to $M$. We then find

$$
\underline{T}_{\alpha \beta} c^{\prime}+2 h_{(\alpha}{\underline{\gamma^{\prime}}}^{\underline{T}_{\beta) \gamma^{\prime}}}{ }^{c^{\prime}}+h_{\alpha}{ }^{\gamma^{\prime}} h_{\beta}{\underline{\delta^{\prime}}}_{\gamma_{\gamma^{\prime} \delta^{\prime}}}^{c^{\prime}}=0
$$

and

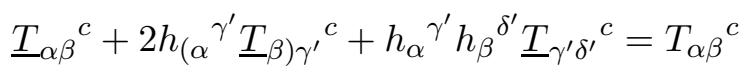

where

$$
\underline{T}_{\alpha \beta}{ }^{c^{\prime}}=u_{\alpha} \underline{\alpha} u_{\beta} \underline{\beta} T_{\underline{\alpha \beta}} \underline{c} u_{\underline{c}}^{c^{\prime}}
$$

and similarly for the other projections of $T_{\underline{\alpha \beta}}$. In order for there to be embeddings of branes in general we require that these equations be satisfied for arbitrary embeddings passing through a 
given point $p \in \underline{M}$ and furthermore that this should be true for all points of $\underline{M}$. Since we may vary $u$ and $h$ independently this requires

$$
\underline{T}_{\alpha \beta} c^{\prime}=0 .
$$

This can only be satisfied for arbitrary embeddings if the the target space tensor is invariant, i.e. if

$$
T_{\underline{\alpha \beta}} \underline{\underline{c}}=-i\left(\Gamma^{\underline{c}}\right)_{\underline{\alpha \beta}} .
$$

Given this one finds that equations (2.12) and (2.13) are solved by (in the case of the supermembrane)

$$
h_{\alpha}^{\beta^{\prime}}=0
$$

while the worldvolume Frobenius tensor is

$$
T_{\alpha \beta}^{c}=-i\left(\Gamma^{c}\right)_{\alpha \beta} .
$$

As an aside we note that, in the case of the 5 -brane, $h$ is no longer zero. It is given by

$$
h_{\alpha}^{\beta^{\prime}}=\frac{1}{6}\left(\Gamma^{a b c}\right)_{\alpha}^{\beta^{\prime}} h_{a b c}
$$

where $h_{a b c}$ is self-dual. This field is related to the self-dual 3-form field strength of the worldvolume tensor multiplet. This a general feature: $h_{\alpha}{ }^{\beta^{\prime}}$ is non-vanishing whenever the worldvolume multiplet includes a gauge field.

To summarise, in this subsection we have shown that the existence of supermembranes as embedded submanifolds through arbitrary points of the (11|32)-dimensional target superspace $\underline{M}$ implies that the equations of motion of eleven-dimensional supergravity must be satisfied. Moreover, the embedding condition determines the worldvolume multiplet to be the on-shell $d=3, N=8$ scalar multiplet, as we shall describe below. Thus the worldvolume and spacetime dynamics are determined by the same simple embedding condition. This argument is thus parallel to that given in the component formalism where it is known that kappa-symmetry implies that the super target space must be a solution of supergravity $[1,5]$.

\subsection{Linearised embeddings}

In local coordinates, $z=(x, \theta)$ for $M$ and $\underline{z}=(\underline{x}, \underline{\theta})$ for $\underline{M}$, the embedding is given as $\underline{z}(z)$. The derivative of the embedding, referred to preferred bases on both spaces, defines what we shall call the embedding matrix, $E_{A} \underline{A}$,

$$
E_{A} \underline{A}=E_{A}{ }^{M} \partial_{M} z^{\underline{M}} E_{\underline{M}}^{\underline{A}},
$$

where $E_{A}{ }^{M}$ is the inverse supervielbein on the worldvolume. In order to get a feel for the components of the embedding matrix consider the case of a flat target space, 


$$
\begin{aligned}
& E^{\underline{a}}=d x^{\underline{a}}-\frac{i}{2} d \theta^{\underline{\alpha}}\left(\Gamma^{\underline{a}}\right)_{\underline{\alpha \beta}} \theta^{\underline{\beta}}, \\
& E^{\underline{\alpha}}=d \theta^{\underline{\alpha}},
\end{aligned}
$$

choose the physical gauge,

$$
\begin{aligned}
& x^{\underline{a}}=\left\{\begin{array}{l}
x^{a} \\
x^{a^{\prime}}(x, \theta)
\end{array}\right. \\
& \theta^{\underline{\alpha}}=\left\{\begin{array}{l}
\theta^{\alpha} \\
\theta^{\alpha^{\prime}}
\end{array}\right.
\end{aligned}
$$

and take the embedding to be infinitesimal so that $E_{A}{ }^{M} \partial_{M}$ can be replaced by $D_{A}=\left(\partial_{a}, D_{\alpha}\right)$ where $D_{\alpha}$ is the flat superspace covariant derivative on the worldvolume. In this limit the embedding condition becomes

$$
D_{\alpha} X^{a^{\prime}}=i\left(\Gamma^{a^{\prime}}\right)_{\alpha \beta^{\prime}} \theta^{\beta^{\prime}}
$$

where

$$
X^{a^{\prime}}:=x^{a^{\prime}}+\frac{i}{2} \theta^{\alpha}\left(\Gamma^{a^{\prime}}\right)_{\alpha \beta^{\prime}} \theta^{\beta^{\prime}} .
$$

One also finds

$$
\begin{gathered}
E_{a} \underline{\underline{b}} \rightarrow\left\{\begin{array}{l}
\delta_{a}{ }^{b} \\
\partial_{a} X^{b^{\prime}}
\end{array}\right. \\
E_{a} \underline{\underline{\beta}} \rightarrow\left\{\begin{array}{l}
0 \\
\partial_{a} \theta^{\beta^{\prime}}
\end{array}\right. \\
E_{\alpha} \underline{\beta} \rightarrow\left\{\begin{array}{l}
\delta_{\alpha}{ }^{\beta} \\
D_{\alpha} \theta^{\beta^{\prime}}
\end{array}\right.
\end{gathered}
$$

One can therefore think of the leading components of $E_{a} \underline{a}$ and $E_{a} \underline{\underline{\alpha}}$ as being the spacetime derivatives of the world surface scalar and spinor fields (i.e. $X^{a^{\prime}}$ and $\theta^{\alpha^{\prime}}$ in the linearised case). From equation (2.23) we find, by differentiating, that

$$
D_{\alpha} \theta^{\beta^{\prime}}=\frac{1}{2}\left(\Gamma^{a b^{\prime}}\right)_{\alpha}^{\beta^{\prime}} \partial_{a} X_{b^{\prime}}
$$

This shows that the only independent components of the Goldstone superfield $X^{a^{\prime}}$ are the eight scalars given by $X^{a^{\prime}} \mid$ and $\theta^{\alpha^{\prime}} \mid$ where the vertical bar denotes the evaluation of a superfield at $\theta=0$. The worldvolume multiplet of the linearised superembedding is therefore the free $d=3, N=8$ on-shell scalar multiplet.

It is instructive to consider this type of embedding to next order in the transverse coordinates $\left(x^{a^{\prime}}, \theta^{\alpha^{\prime}}\right)$. We can write 


$$
E_{A}=D_{A}-H_{A}^{B} D_{B}
$$

where $H_{A}{ }^{B}$ is the linearised supervielbein. To first order in the transverse coordinates it is easy to show that $H$ vanishes (in the physical gauge), but at second order one finds, from (2.5)

$$
H_{\alpha}{ }^{b}=-\frac{i}{2} D_{\alpha} \theta^{\beta^{\prime}}\left(\Gamma^{b}\right)_{\beta^{\prime} \gamma^{\prime}} \theta^{\gamma^{\prime}}
$$

Since one can determine the remaining components of $H_{A}{ }^{B}$ from the constraints on the worldvolume torsion, this shows that the induced geometry of the worldvolume is indeed determined by the embedding as claimed.

\section{Lorentzian supergeometry and superembeddings}

\subsection{Target superspace geometry}

The discussion of the previous section makes no reference to connections, torsion or curvature, but in order to interpret the equations given there it is convenient to introduce these objects in order to obtain manifestly covariant equations of motion for the component fields. Our approach is similar to that of [13], although we work in an arbitrary supergravity background and give more details of the equations of motion and the induced supergeometry on the brane. We begin with the target space $\underline{M}$. We first make a choice of the even tangent bundle $\underline{B}$ (i.e. fix $\left(E_{a}\right)$ ) which reduces the structure group to $\operatorname{Spin}(1,10) \times \mathbb{R}^{+}:=C \operatorname{Spin}(1,10)$. We then introduce a connection, $\underline{\Omega}$, but only for $\operatorname{Spin}(1,10)$, since the scale part of the group can be dealt with separately. We define the torsion and curvature 2 -forms as usual by

$$
\begin{aligned}
T^{\underline{A}} & =d E^{\underline{A}}+E^{\underline{B}} \Omega_{\underline{B}} \underline{A} \\
R_{\underline{A}} \underline{B} & =d \Omega_{\underline{A}} \underline{B}+\Omega_{\underline{A}} \underline{C} \Omega_{\underline{C}} \underline{B},
\end{aligned}
$$

where the basis forms $\left(E^{\underline{A}}\right)$ are related to the coordinate basis by the vielbein matrix, $E_{\underline{M}} \underline{A}$,

$$
E^{\underline{A}}=d z^{\underline{M}} E_{\underline{M}} \underline{A}
$$

whose inverse is denoted $E_{\underline{A}} \underline{M}$.

The dimension zero component of the torsion tensor is identified with the Frobenius tensor of the last section and is thus required to be, if embeddings of branes are to be permitted,

$$
T_{\underline{\alpha \beta}}^{\underline{c}}=-i\left(\Gamma^{\underline{c}}\right)_{\underline{\alpha \beta}} .
$$

The dimension one-half component of the connection and $E_{\underline{a}}$ can be chosen such that almost all components of the dimension one-half torsion vanish except for a single spinor field. However, it can be shown that this spinor is the odd derivative of a scalar superfield, at least provided that $\underline{M}$ is simply connected, and that this scalar field can be transformed away by a super-Weyl 
transformation. We shall assume throughout the rest of this paper that this manoeuvre is both allowable and has been carried out so that

$$
T_{\underline{\alpha} \underline{\underline{c}}}^{\underline{ }}=T_{\underline{\alpha \beta}}^{\underline{\gamma}}=0 \text {. }
$$

At dimension one one may impose

$$
T_{\underline{a b}} \underline{c}=0
$$

in which case one finds

$$
T_{\underline{a \beta}} \underline{\underline{\gamma}}=-\frac{1}{36}(\Gamma \underline{b c d})_{\underline{\beta}} \underline{\underline{\gamma}} G_{\underline{a b c d}}-\frac{1}{288}\left(\Gamma_{\underline{a b c d e}}\right)_{\underline{\beta}} \underline{\underline{ }} G \underline{b c d e} .
$$

The dimension three-halves torsion, whose leading component in the $\underline{\theta}$-expansion is the field strength tensor for the gravitino field, is given as the odd derivative of the field $G$. The curvature can be computed in terms of the torsion, i.e. in terms of $G$ and its derivatives, by virtue of Dragon's theorem which is applicable in this case. Finally, given the above, one can establish the existence of a closed superspace four-form $G_{4}$ whose non-vanishing components are $G_{\underline{a b c d}}$ and

$$
G_{\underline{a b \gamma \delta}}=-i\left(\Gamma_{\underline{a b}}\right)_{\underline{\gamma} \underline{\delta}} .
$$

\subsection{Superembeddings}

The bosonic degrees of freedom are all scalar. In the context of superembeddings this implies the vanishing of the field $h_{\alpha}{ }^{\beta^{\prime}}$ as we saw previously, so that

$$
E_{\alpha} \underline{\underline{\alpha}}=u_{\alpha}^{\underline{\alpha}}, \quad T_{\alpha \beta}^{c}=-i\left(\Gamma^{c}\right)_{\alpha \beta} .
$$

The first task is to specify the various bundles that arise. This is more complicated than the Riemannian case where one has only to fix the normal bundle which can be done by choosing it to be the orthogonal complement of the tangent bundle of the surface. In the supersymmetric case we have the additional complication of even and odd bundles, so that there are nine bundles in all to think about; odd, even and total for the target space, the worldvolume and the normal direction to the worldvolume. We shall assume that the geometry of the target space is standard so that a decomposition $\underline{T}=\underline{F} \oplus \underline{B}$ for the target space bundles has been chosen such that equations (3.4) hold. It therefore remains to specify how $\underline{T}$ decomposes in terms of tangential and normal components.

It is natural to take the odd normal bundle $F^{\prime}$ to be a subbundle of $\underline{F}$ complementary to $F$. Dually, one can take $B^{* *}$ to be a subbundle of $\underline{B}^{*}$ complementary to $B^{*}$. We introduce a basis $\left(E_{A^{\prime}}\right)=\left(E_{a^{\prime}}, E_{\alpha^{\prime}}\right)$ for the normal bundle $T^{\prime}$ related to the preferred basis for the target space by the normal matrix, $E_{A^{\prime}}$,

$$
E_{A^{\prime}}=E_{A^{\prime}} \underline{A} E_{\underline{A}},
$$


The assumption that $F^{\prime}$ is a subspace of $\underline{F}$ is equivalent to

$$
E_{\alpha^{\prime}} \stackrel{a}{=}=0
$$

$F^{\prime}$ and $B^{\prime *}$ can be specified completely using the Frobenius tensor. We may choose

$$
\begin{aligned}
E_{\alpha} \underline{\alpha} & =u_{\alpha} \underline{\alpha} \\
E_{\alpha^{\prime}} \underline{\alpha} & =u_{\alpha^{\prime}} \underline{\alpha}
\end{aligned}
$$

and

$$
\begin{aligned}
E_{a} \underline{a} & =u_{a^{\underline{a}}}^{\underline{a}} \\
E_{a^{\prime}} \underline{a} & =u_{a^{\prime}} \underline{a}
\end{aligned}
$$

where $\left(u \underline{\alpha}, u_{\alpha^{\prime}} \underline{\alpha}\right)$ is an element of $\operatorname{Spin}(1,10)$ and $\left(u_{a^{a}} \underline{\underline{a}}, u_{a^{\prime}} \underline{a}\right)$ is the corresponding element of the Lorentz group. This is accomplished by demanding that the Frobenius tensor decompose as follows

$$
T_{\underline{\alpha \beta}} \underline{c} \rightarrow \begin{cases}T_{\alpha \beta}{ }^{c} & =-i\left(\Gamma^{c}\right)_{\alpha \beta} \\ T_{\alpha^{\prime} \beta^{\prime}}{ }^{c} & =-i\left(\Gamma^{c}\right)_{\alpha^{\prime} \beta^{\prime}} \\ T_{\alpha \beta^{\prime}} c^{\prime} & =-i\left(\Gamma^{c^{\prime}}\right)_{\alpha \beta^{\prime}}=T_{\beta^{\prime} \alpha} c^{\prime}\end{cases}
$$

with all other components of $T_{\underline{\alpha} \beta} \underline{c}$ being zero. Since we have a Lorentzian metric on $\underline{B}$, the conformal factor allowed by considerations of the Frobenius tensor alone can be set equal to one. This is implicit in the expressions for $E_{a} \underline{a}$ and $E_{a^{\prime}} \underline{a}$ given above. Equivalently we could impose

$$
E_{a} \underline{\underline{a}} E_{b} \underline{\underline{b}} \eta_{\underline{a b}}=\eta_{a b}
$$

and

$$
E_{a^{\prime}} \underline{a} E_{b^{\prime}} \underline{b} \eta_{\underline{a b}}=\delta_{a^{\prime} b^{\prime}}
$$

The embedding matrix together with the normal matrix makes up a square matrix with entries $\left(E_{A^{\underline{A}}}, E_{A^{\prime}} \underline{A}\right)$ whose inverse will be denoted by $\left(\left(E^{-1}\right)_{\underline{A}}^{A},\left(E^{-1} \underline{\underline{A}}^{A^{\prime}}\right)\right.$. In view of the embedding condition (2.5) one finds that $\left(\left(E^{-1}\right)_{\underline{\alpha}}{ }^{\alpha},\left(E^{-1}\right)_{\underline{\alpha}}{ }^{\alpha^{\prime}}\right)$ is the inverse of $\left(E_{\alpha} \underline{\alpha}, E_{\alpha^{\prime}} \underline{\alpha}\right)$ and that $\left(\left(E^{-1}\right)_{\underline{a}}^{a},\left(E^{-1}\right)_{\underline{a}}^{a^{\prime}}\right)$ is the inverse of $\left(E_{a} \underline{a}, E_{a^{\prime}} \underline{a}\right)$. Furthermore,

$$
\left(E^{-1}\right)_{\underline{\alpha}}^{a}=\left(E^{-1} \underline{\underline{\alpha}}^{a^{\prime}}=0 .\right.
$$

The inverse is completed by

$$
\begin{aligned}
& \left(E^{-1}\right)_{\underline{a}}^{\alpha}=\left(E^{-1}\right)_{\underline{a}}{ }^{a} E_{a} \underline{\alpha}\left(E^{-1}\right)_{\underline{\alpha}}^{\alpha}+\left(E^{-1}\right)_{\underline{a}}^{a^{\prime}} E_{a^{\prime}}{ }^{\alpha}\left(E^{-1}\right)_{\underline{\alpha}}{ }^{\alpha} \\
& \left(E^{-1}\right)_{\underline{a}}^{\alpha^{\prime}}=\left(E^{-1}\right)_{\underline{a}}^{a} E_{a} \underline{\alpha}\left(E^{-1}\right)_{\underline{\alpha}}^{\alpha^{\prime}}+\left(E^{-1}\right)_{\underline{a}}^{a^{\prime}} E_{a^{\prime}}{ }^{\underline{\alpha}}\left(E^{-1}\right)_{\underline{\alpha}}^{\alpha^{\prime}} \text {. }
\end{aligned}
$$


The embedding condition does not fully specify the even tangent bundle $B$ of $M$. Making a choice of $B$ is equivalent to choosing a basis set of even vectors, $\left(E_{a}\right)$, which can be modified by

$$
E_{a} \mapsto E_{a}+\rho_{a}{ }^{\alpha} E_{\alpha}
$$

¿From an embedding point of view one has

$$
E_{a}=E_{a} \underline{a} E_{\underline{a}}+E_{a} \underline{\alpha} E_{\underline{\alpha}} .
$$

The second term can be written as

$$
E_{a} \underline{\alpha} E_{\underline{\alpha}}=E_{a} \underline{\underline{\alpha}}\left(E^{-1}\right)_{\underline{\alpha}}^{\alpha} E_{\alpha}+E_{a} \underline{\underline{\alpha}}\left(E^{-1}\right)_{\underline{\alpha}}^{\alpha^{\prime}} E_{\alpha^{\prime}} .
$$

By using a redefinition of the form (3.18), one can clearly choose $\rho$ so that the first term on the right in (3.20) is removed. Thus we can always make the standard choice of $B$ for which

$$
E_{a} \underline{\underline{\alpha}}=\Lambda_{a}^{\alpha^{\prime}} E_{\alpha^{\prime}} \underline{\alpha}
$$

Finally we can choose $B^{\prime}$ to be the orthogonal complement of $B \cap \underline{B}$ and to have vanishing projection on $\underline{F}$.

In summary, we may choose the basis vectors for the spaces $F, B, F^{\prime}$ and $B^{\prime}$ by specifying the components of the embedding and normal matrices to be

$$
\begin{array}{ll}
E_{a} \underline{a}=u_{a} \underline{a} & E_{a} \underline{\alpha}=\Lambda_{a}^{\alpha^{\prime}} u_{\alpha^{\prime}} \underline{\alpha} \\
E_{\alpha} \underline{a}=0 & E_{\alpha} \underline{\alpha}=u_{\alpha} \underline{\alpha}
\end{array}
$$

and

$$
\begin{array}{ll}
E_{a^{\prime}} \underline{a}=u_{a^{\prime}} \underline{a} & E_{a^{\prime}} \underline{\alpha}=0 \\
E_{\alpha^{\prime}} \underline{a}=0 & E_{\alpha^{\prime}} \underline{\alpha}=u_{\alpha^{\prime}} \underline{\alpha}
\end{array}
$$

respectively. One can then compute the components of the inverse matrices to be

$$
\begin{array}{ll}
\left(E^{-1}\right)_{\underline{a}}{ }^{a}=u_{\underline{a}}{ }^{a} & \left(E^{-1}\right)_{\underline{a}^{\alpha}}=0 \\
\left(E^{-1}\right)_{\underline{\alpha}}^{a}=0 & \left(E^{-1}\right)_{\underline{\alpha}}^{\alpha}=u_{\underline{\alpha}^{\alpha}}{ }^{\alpha}
\end{array}
$$

and

$$
\begin{array}{lll}
\left(E^{-1}\right)_{\underline{a}}^{a^{\prime}}=u_{\underline{a}}{ }^{a^{\prime}} & \left(E^{-1}\right)_{\underline{a}}{ }^{\alpha^{\prime}}=-u_{\underline{a}}{ }^{a} \Lambda_{a}{ }^{\alpha^{\prime}} \\
\left(E^{-1}\right)_{\underline{\alpha}} \alpha^{\prime}=u_{\underline{\alpha}} & \left(E^{-1}\right)_{\underline{\alpha}} a^{\prime}=0 .
\end{array}
$$

The structure groups induced on the worldvolume bundles by the embedding are $\operatorname{Spin}(1,2)$. $\operatorname{Spin}(8)$ on $F$ and $F^{\prime}$ (but acting via the two inequivalent minimal representations of Spin(8)), $S O_{o}(1,2)$ on $B$ (with the superscript "o" denoting the component connected to the identity) and $S O(8)$ on $B^{\prime}$, the orthogonal groups being related to the spin groups by the $2: 1$ maps determined by the $\Gamma$-matrices. 
We shall now turn to the induced connection on the worldvolume. This can be specified by means of the superspace version of the Gauss-Weingarten equations which were discussed in [13]. These are summarised in the Riemannian case in (B.6) in the appendix. The super Gauss-Weingarten equations are

$$
\begin{aligned}
\nabla_{A} E_{b^{\underline{c}}} & =K_{A, b}{ }^{c^{\prime}} E_{c^{\prime}} \underline{c} \\
\nabla_{A} E_{b^{\prime}} \underline{\underline{c}} & =K_{A, b^{\prime}}{ }^{c} E_{c} \underline{\underline{c}}
\end{aligned}
$$

and

$$
\begin{aligned}
\nabla_{A} E_{\beta} \underline{\underline{ }} & =K_{A, \beta^{\gamma^{\prime}}} E_{\gamma^{\prime}} \underline{\underline{ }} \\
\nabla_{A} E_{\beta^{\prime}} \underline{\underline{y}} & =K_{A, \beta^{\prime}} E_{\gamma^{\gamma}}
\end{aligned}
$$

where

$$
\begin{aligned}
& \nabla_{A} E_{b} \underline{\underline{c}}=E_{A} E_{b} \underline{\underline{c}}-\Omega_{A, b}{ }^{c} E_{c} \underline{\underline{c}}+E_{b} \underline{\underline{b}} \Omega_{A, \underline{b}} \underline{\underline{c}} \\
& \nabla_{A} E_{b^{\prime}} \underline{\underline{c}}=E_{A} E_{b^{\prime}} \underline{c}-\Omega_{A, b^{\prime}} c^{\prime} E_{c^{\prime}} \underline{c}+E_{b^{\prime}} \underline{\underline{b}} \Omega_{A, \underline{b}} \underline{c} \\
& \nabla_{A} E_{\beta} \underline{\underline{\gamma}}=E_{A} E_{\beta} \underline{\underline{\gamma}}-\Omega_{A, \beta} E_{\gamma} \underline{\underline{\gamma}}+E_{\beta} \underline{\beta} \Omega_{A, \underline{\beta}} \underline{\underline{\gamma}} \\
& \nabla_{A} E_{\beta^{\prime}} \underline{\underline{q}}=E_{A} E_{\beta^{\prime}} \underline{\underline{c}}-\Omega_{A, \beta^{\prime}}{\underline{\gamma^{\prime}}}^{E_{\gamma^{\prime}} \underline{\underline{ }}}+E_{\beta^{\prime}} \underline{\beta} \Omega_{A, \underline{\beta}} \underline{\underline{q}} \text {. }
\end{aligned}
$$

In these equations $\Omega_{A, b}{ }^{c}$, etc. are connections on the worldvolume while $\Omega_{A, \underline{b}} \underline{c}$ and $\Omega_{A, \underline{\beta}} \underline{\underline{q}}$ are the pull-backs of the target space connections in $\underline{B}$ and $\underline{F}$. For example,

$$
\Omega_{A, \underline{\underline{c}}}^{\underline{c}}=E_{A} \underline{A}_{\underline{A}, \underline{\underline{b}}}^{\underline{c}} .
$$

Note that the Gauss-Weingarten equations as defined above differ slightly from the Riemannian case in that not all components of the embedding matrix appear. Indeed, if $Y$ is an even vector on $M, Y^{a} E_{a} \underline{a}$ does not give the components of $Y$ considered as a vector on $\underline{M}$, but only the projection of this vector onto $\underline{B}$.

Because of the $\operatorname{Spin}(1,10)$ structure on the target space we have

$$
\Omega_{A, \underline{\beta}} \underline{\mathcal{Y}}=\frac{1}{4}\left(\Gamma^{\underline{b c}}\right)_{\underline{\underline{\beta}}}{ }^{\underline{\gamma}} \Omega_{A, \underline{b c}} .
$$

It is not difficult to show that the induced connections preserve the various tensors that are induced on the worldvolume bundles from the metrics and the Frobenius tensor on $M$, so that one has the following relations between the connections

$$
\begin{aligned}
\Omega_{A, b c} & =-\Omega_{A, c b} \\
\Omega_{A, b^{\prime} c^{\prime}} & =-\Omega_{A, c^{\prime} b^{\prime}}
\end{aligned}
$$




$$
\begin{aligned}
\Omega_{A, \beta}{ }^{\gamma} & =\frac{1}{4}\left(\Gamma^{b c}\right)_{\beta}{ }^{\gamma} \Omega_{A, b c}+\frac{1}{4}\left(\Gamma^{b^{\prime} c^{\prime}}\right)_{\beta^{\gamma}} \Omega_{A, b^{\prime} c^{\prime}} \\
\Omega_{A, \beta^{\prime}} \gamma^{\prime} & =\frac{1}{4}\left(\Gamma^{b c}\right)_{\beta^{\prime}} \gamma^{\prime} \Omega_{A, b c}+\frac{1}{4}\left(\Gamma^{b^{\prime} c^{\prime}}\right)_{\beta^{\prime}} \gamma^{\prime} \Omega_{A, b^{\prime} c^{\prime}}
\end{aligned}
$$

while the components of the second fundamental form obey

$$
\begin{aligned}
K_{A b, c^{\prime}} & =-K_{A, c^{\prime}, b} \\
K_{A, \beta} \gamma^{\prime} & =\frac{1}{2}\left(\Gamma^{b c^{\prime}}\right)_{\beta} \gamma^{\prime} K_{A, b c^{\prime}} \\
K_{A, \beta^{\prime}} \gamma & =\frac{1}{2}\left(\Gamma^{b c^{\prime}}\right)_{\beta^{\prime}}{ }^{\gamma} K_{A, b c^{\prime}} .
\end{aligned}
$$

The connections can equally well be defined from the $\mathfrak{s p i n}(1,10)$-valued one-form

$$
K_{A}=\left(\nabla_{A} u\right) u^{-1}
$$

where $u=\left(u_{\alpha} \underline{\underline{\alpha}}, u_{\alpha^{\prime}} \underline{\underline{\alpha}}\right)$, and where $\nabla_{A}$ acts on $u$ as in (3.28). The 1 -form $K$ can be defined for any worldvolume connection; the induced connection is given by demanding that the $\mathfrak{s p i n}(1,2)$ and $\mathfrak{s p i n}(8)$ components vanish. The remaining non-zero components then satisfy equations (3.32).

The equation defining the target space torsion 2-form, pulled back to the worldvolume, reads

$$
\nabla_{A} E_{B} \underline{C}-(-1)^{A B} \nabla_{B} E_{A} \underline{\underline{C}}+T_{A B}^{C} E_{C} \underline{C}=(-1)^{(A(B+\underline{B})} E_{B} \underline{\underline{B}} E_{A} \underline{\underline{A}} T_{\underline{A B}} \underline{\underline{C}} .
$$

Finally, by differentiating (3.33), we obtain

$$
\nabla_{A} K_{B}-\nabla_{B} K_{A}+T_{A B}^{C} K_{C}-\left[K_{A}, K_{B}\right]=\left(R_{A B} u\right) u^{-1}
$$

where the curvature form acts on the indices of $u$ according to the prescription given in (3.28). For the case of induced connections, one finds the equations of Gauss and Codazzi,

$$
\begin{aligned}
R_{A B, c}{ }^{d} & =\underline{R}_{A B, c}{ }^{d}+\left(K_{A, c^{e^{\prime}}} K_{B, e^{\prime}}{ }^{d} \mp(A \leftrightarrow B)\right) \\
R_{A B, c^{\prime}}{ }^{d^{\prime}} & =\underline{R}_{A B, c^{\prime}}{ }^{d^{\prime}}+\left(K_{A, c}{ }^{e} K_{B, e}{ }^{d^{\prime}} \mp(A \leftrightarrow B)\right)
\end{aligned}
$$

where

$$
\underline{R}_{A B, c}{ }^{d}=(-1)^{A(B+\underline{B})} E_{B} \underline{\underline{B}} E_{A} \underline{A} u_{c} \underline{\underline{c}} R_{\underline{A B}, \underline{c}} \underline{\underline{d}} u_{\underline{d}}{ }^{d}
$$

and, similarly,

$$
\underline{R}_{A B, c^{\prime}}{ }^{d^{\prime}}=(-1)^{A(B+\underline{B})} E_{B} \underline{B} E_{A} \underline{A}_{u_{c^{\prime}}} R_{\underline{A B}, \underline{c} \underline{\underline{d}}} u_{\underline{d}}^{d^{\prime}} .
$$


The Gauss-Codazzi equations given above are enough to define all of the curvature components because those with spinor indices are determined by those with vector indices according to the relations (3.31) which hold also for the curvature forms.

The torsion equation (3.34) can be used to determine the induced worldvolume torsion as well as the constraints satisfied by the superfield $\Lambda$. The curvature components can be read off using the Gauss-Codazzi equation.

\section{The dynamics of the M2-brane}

\subsection{The torsion equations}

In this section we analyse the membrane embedding by systematically going through the torsion equation (3.34). We shall take the worldvolume connection to be induced and the embedding to be of the form given in equations (3.22) and (3.23). The analysis will determine the worldvolume torsion and the equations of motion of the membrane in an arbitrary supergravity background. The worldvolume curvature can be found using the Gauss-Codazzi equations.

In natural units $E_{\alpha} \underline{\underline{\alpha}}$ and $E_{a} \underline{\underline{a}}$ have dimension zero, $E_{\alpha} \underline{\underline{a}}$ has dimension minus one-half and $E_{a} \underline{\underline{\alpha}}$ has dimension plus one-half. The dimension zero component of the torsion equation simply reduces to the relation (2.8) between the Frobenius tensors of the worldvolume and the target space, and this has already been taken account of in writing down the form of the embedding matrix (3.22).

At dimension one-half one has two equations,

$$
\nabla_{\alpha} E_{\beta} \underline{\underline{q}}+\nabla_{\beta} E_{\alpha} \underline{\underline{ }}+T_{\alpha \beta}^{c} E_{c} \underline{\underline{\gamma}}+T_{\alpha \beta}^{\gamma} E_{\gamma} \underline{\underline{ }}=0
$$

and

$$
\nabla_{\alpha} E_{b} \underline{c}+T_{\alpha b}^{c} E_{c} \underline{c}=-E_{b} \underline{\beta} E_{\alpha} \underline{\alpha} T_{\alpha \underline{\beta}}^{\underline{c}}=i E_{b} \underline{\beta} E_{\alpha} \underline{\alpha}\left(\Gamma^{\underline{c}}\right)_{\underline{\alpha \beta}}
$$

where the vanishing of $E_{\alpha} \underline{a}$ has been taken into account. These can be analysed by rightmultiplying by $u^{-1}$ in the appropriate representation. Right-multiplying (4.1) by $u_{\chi^{\gamma}}^{\gamma}$ one finds

$$
T_{\alpha \beta}^{\gamma}=0
$$

where we have used the induced connection condition $K_{\alpha, \beta} \gamma=0$ and the embedding choice (3.21). Similarly, right-multiplying (4.2) by $u_{\underline{c}}^{c}$, one finds

$$
T_{\alpha b}^{c}=0
$$

Right-multiplying (4.2) with $u_{\underline{c}}^{c^{\prime}}$ gives

$$
K_{\alpha, b c^{\prime}}=\left(\Gamma_{c^{\prime}}\right)_{\alpha \beta^{\prime}} \Lambda_{b}{ }^{\beta^{\prime}}
$$

from which one can deduce, using (3.32), that 


$$
K_{\alpha, \beta}^{\gamma^{\prime}}=\frac{i}{2}\left(\Gamma^{a b^{\prime}}\right)_{\beta}^{\gamma^{\prime}}\left(\Gamma_{b^{\prime}}\right)_{\alpha \delta^{\prime}} \Lambda_{a}^{\delta^{\prime}}
$$

The remaining dimension one-half equation is found by right-multiplying (4.1) by $u_{\underline{\Upsilon}}{ }^{\gamma^{\prime}}$. This gives

$$
K_{\alpha, \beta}^{\gamma^{\prime}}+K_{\beta, \alpha}^{\gamma^{\prime}}=i\left(\Gamma^{c}\right)_{\alpha \beta} \Lambda_{c}^{\gamma^{\prime}}
$$

Due to (4.6) this relation involves only the field $\Lambda_{a}{ }^{\alpha^{\prime}}$; it is satisfied provided that

$$
\left(\Gamma^{a}\right)_{\alpha \beta^{\prime}} \Lambda_{a}^{\beta^{\prime}}=0
$$

In view of the linearised analysis, one sees that the leading component of this equation is the Dirac equation for the worldvolume fermion field.

There are also two equations at dimension one:

$$
\nabla_{a} E_{\beta} \underline{\underline{\gamma}}-\nabla_{\beta} E_{a} \underline{\underline{\gamma}}+T_{a \beta}{ }^{\gamma} E_{\gamma} \underline{\underline{\gamma}}=E_{\beta} \underline{\underline{\beta}} E_{a} \underline{\underline{a}} T_{\underline{a} \beta} \underline{\underline{c}}=0
$$

and

$$
\nabla_{a} E_{b}^{\underline{\underline{c}}}-\nabla_{b} E_{a}^{\underline{\underline{c}}}+T_{a b}^{c} E_{c}^{\underline{\underline{c}}}=E_{b} \underline{\underline{\beta}} E_{a} \underline{\underline{\alpha}} T_{\underline{\alpha} \underline{\beta}}^{\underline{a}} .
$$

Right-multiplying by $u$ as above one finds, from (4.9),

$$
\begin{aligned}
T_{a \beta}{ }^{\gamma} & =\underline{T}_{a \beta}{ }^{\gamma}-\Lambda_{a}^{\delta^{\prime}} K_{\beta, \delta^{\prime}} \gamma \\
K_{a, \beta} \gamma^{\prime}-\nabla_{\beta} \Lambda_{a}^{\gamma^{\prime}} & =\underline{T}_{a \beta} \gamma^{\prime}
\end{aligned}
$$

¿From (4.10) one finds

$$
\begin{aligned}
T_{a b}^{c} & =i \Lambda_{a}^{\alpha^{\prime}}\left(\Gamma^{c}\right)_{\alpha^{\prime} \beta^{\prime}} \Lambda_{b}^{\beta^{\prime}} \\
K_{a, b} c^{\prime} & =K_{b, a} c^{\prime} .
\end{aligned}
$$

The first and third of these equations determine the dimension one components of the worldvolume torsion while the fourth shows that the bosonic part of the second fundamental form is symmetric even though $T_{a b}{ }^{c}$ is not zero. The main equation to analyse is therefore the second one. To do this we shall switch to the two step notation (see appendix) in which worldvolume spinor indices are explicitly decomposed into representations of $S L(2, \mathbb{R}) \times O(8)$. We may set

$$
\nabla_{\beta} \Lambda_{a}^{\gamma^{\prime}} \rightarrow \nabla_{\beta j} \Lambda_{a}{ }^{\gamma k^{\prime}}=\delta_{\beta}{ }^{\gamma} M_{a j}{ }^{\prime}+\left(\gamma^{b}\right)_{\beta}{ }^{\gamma} N_{a b j}{ }^{\prime}
$$

The Dirac equation for $\Lambda$ implies 


$$
\begin{aligned}
\eta^{a b} N_{a b j k^{\prime}} & =0 \\
M_{a j k^{\prime}} & =\epsilon_{a b c} N_{j k^{\prime}}^{b c}
\end{aligned}
$$

Substituting for $\nabla \Lambda$ in terms of $M$ and $N$ in (4.12) one finds the following further conditions

$$
\begin{aligned}
N_{(a b) j k^{\prime}} & =\frac{1}{2}\left(\sigma^{c^{\prime}}\right)_{j k^{\prime}}\left(K_{a b, c^{\prime}}-\frac{1}{3} \eta_{a b} \eta^{c d} K_{c d, c^{\prime}}\right) \\
M_{a, j k^{\prime}} & =+\frac{1}{36}\left(\sigma^{b^{\prime} c^{\prime} d^{\prime}}\right)_{j k^{\prime}} \underline{G}_{a b^{\prime} c^{\prime} d^{\prime}}
\end{aligned}
$$

as well as

$$
\eta^{a b} K_{a b, d^{\prime}}=-\frac{1}{6} \epsilon^{a b c} \underline{G}_{a b c}{ }^{d^{\prime}}
$$

Since $K_{a, b} c^{\prime}$ is essentially the second derivative of the worldvolume scalar field, these equations show that all dimension one quantities are determined in terms of known fields and their derivatives. Equation (4.18), or rather its leading component, is the scalar equation of motion. Using the fact that $K_{a, b c}=0$, the definition of $K$ and noting that $\underline{G}_{a b c d}=0$ identically, one can rewrite it in the form

$$
\eta^{a b} \nabla_{a} E_{b} \underline{\underline{d}}=-\frac{1}{6} \epsilon^{a b c} \underline{G}_{a b c} \underline{\underline{d}} .
$$

This is the scalar equation in compact form in the presence of a supergravity background. We remind the reader that, throughout this section, we have used the notation that underlined tensors with non-underlined indices are obtained from the target space tensors by projecting with suitable factors of the $u$ matrices.

There is one equation at dimension three-halves which can be used to determine the dimension three-halves worldvolume torsion component. Finally, one can use the Gauss-Codazzi equations (3.36) to determine the worldvolume curvature directly.

\subsection{The equations of motion in component form}

In order to derive the component form of the equations of motion it is necessary to relate the components of the worldvolume supervielbein to known quantities, at least to leading order in $\theta$. It is always possible to choose a gauge in which the supervielbein takes the form

$$
\begin{aligned}
& E_{m}{ }^{a}(x, \theta)\left|=e_{m}{ }^{a}(x) \quad E_{m}{ }^{\alpha}(x, \theta)\right|=\psi_{m}{ }^{\alpha}(x) \\
& E_{\mu}{ }^{a}(x, \theta)\left|=0 \quad E_{\mu}{ }^{\alpha}(x, \theta)\right|=\delta_{\mu}{ }^{\alpha},
\end{aligned}
$$

where the vertical bar indicates evaluation at $\theta=0$. The inverse supervielbein to leading order is given by 


$$
\begin{aligned}
E_{a}{ }^{m}(x, \theta) \mid & =e_{a}{ }^{m}(x) & E_{a}{ }^{\mu}(x, \theta) \mid & =-\psi_{a}{ }^{\mu}(x) \\
E_{\alpha}{ }^{m}(x, \theta) \mid & =0 & E_{\alpha}{ }^{\mu}(x, \theta) \mid & =\delta_{\alpha}{ }^{\mu},
\end{aligned}
$$

where

$$
\psi_{a}^{\mu}=e_{a}^{m} \psi_{m}{ }^{\alpha} \delta_{\alpha}{ }^{\mu}
$$

For the connection we can choose

$$
\Omega_{m}\left|=\omega_{m}(x), \quad \Omega_{\mu}\right|=0
$$

so that

$$
\Omega_{a}\left|=\omega_{a}:=e_{a}^{m} \omega_{m}, \quad \Omega_{\alpha}\right|=0 .
$$

It is not difficult to see that the worldvolume vielbein $e_{m}{ }^{a}$ is the vielbein associated with the induced Green-Schwarz (GS) metric,

$$
g_{m n}=\mathcal{E}_{m} \underline{\underline{a}} \mathcal{E}_{n} \underline{\underline{b}} \eta_{\underline{a b}}
$$

where

$$
\mathcal{E}_{m} \underline{a}:=\partial_{m} z^{\underline{M}} E_{\underline{M}} \underline{a} \mid .
$$

This is because $E_{\alpha} \underline{a}=0$ implies, (from the definition (2.20)) that

$$
\partial_{\mu} z \underline{\underline{M}} E_{\underline{M}} \underline{\underline{a}} \mid=0
$$

and so

$$
E_{a} \underline{a} \mid=e_{a}^{m} \mathcal{E}_{m} \underline{a} .
$$

Since $E_{a} \underline{a}$ is orthonormal with respect to the target space metric it follows that $e_{m}{ }^{a}$ is indeed the vielbein for the GS metric.

For the worldvolume gravitino we have

$$
\begin{aligned}
\psi_{m}{ }^{\alpha} & =\partial_{m} z^{\underline{M}} E_{\underline{M}} \underline{A} E_{\underline{A}}{ }^{\alpha} \mid \\
& =\mathcal{E}_{m} \underline{\alpha}_{\underline{\alpha}}{ }^{\alpha}
\end{aligned}
$$

where $v=u \mid$ (for any relevant pair of indices), and where

$$
\mathcal{E}_{m} \underline{\underline{\alpha}}:=\partial_{m} z^{\underline{M}} E_{\underline{M}}^{\underline{\alpha}} \mid
$$


This follows from the fact that $E_{\underline{a}}{ }^{\alpha}=0$.

For the embedding matrix, we find, at leading order,

$$
\begin{array}{ll}
E_{a} \underline{\underline{\alpha}} \mid=\mathcal{E}_{a} \underline{\beta} Q_{\underline{\beta}} \underline{\underline{\alpha}} & E_{a} \underline{a} \mid=\mathcal{E}_{a} \underline{a}=v_{a} \underline{\underline{a}} \\
E_{\alpha} \underline{a} \mid=0 & E_{\alpha} \underline{\underline{\alpha}} \mid=v_{\alpha} \underline{\underline{\alpha}}
\end{array}
$$

where

$$
\begin{aligned}
\mathcal{E}_{a} \underline{a} & =e_{a}{ }^{m} \mathcal{E}_{m} \underline{\underline{a}} \\
\mathcal{E}_{a} \underline{\underline{\alpha}} & =e_{a}{ }^{m} \mathcal{E}_{m} \underline{\underline{\alpha}}
\end{aligned}
$$

and where the projection operator $Q$ is given by

$$
Q_{\underline{\alpha}}^{\underline{\beta}}=v_{\underline{\alpha}}^{\gamma^{\prime}} v_{\gamma^{\prime}} \underline{\beta}:=\frac{1}{2}(1-\Gamma) .
$$

We shall denote the complementary projector by $P$,

$$
P_{\underline{\alpha}} \underline{\beta}=v_{\underline{\alpha}}^{\gamma} v_{\gamma} \underline{\beta}=\frac{1}{2}(1+\Gamma)
$$

We next consider the spin one-half equation of motion which is the leading component of (4.8). Consider

$$
E_{a} \underline{\alpha}\left(\Gamma^{a}\right)_{\underline{\alpha \beta}}=\Lambda_{a}^{\alpha^{\prime}}\left(\Gamma^{a}\right)_{\alpha^{\prime} \underline{\beta}}=0
$$

where the last step follows by the equation of motion if $\underline{\beta}=\beta^{\prime}$ and by the properties of the gamma-matrices if $\underline{\beta}=\beta$. Evaluating this at $\theta=0$ and using (4.31) one finds the component spin-half equation

$$
\mathcal{E}_{a} \underline{\beta} Q_{\underline{\beta}}^{\underline{\alpha}}\left(\Gamma^{a}\right)_{\underline{\alpha \gamma}}=0
$$

or, equivalently,

$$
g^{m n} \mathcal{E}_{m} \underline{\underline{ }}(1-\Gamma)_{\underline{\underline{ }}} \underline{\underline{\beta}}\left(\Gamma_{m}\right) \underline{\underline{\alpha}} \underline{\underline{\alpha}}=0
$$

where $g$ is the induced Green-Schwarz metric and $\Gamma_{m}=e_{m}{ }^{a} \Gamma_{a}$.

It is necessary to work a little harder to get the scalar equation of motion out in component form. It is given by the leading component of the superspace equation (4.19). One has

$$
\nabla_{a} E_{b} \underline{\underline{d}}=E_{a}^{m} \partial_{m} E_{b} \underline{\underline{d}}+E_{a}{ }^{\mu} \partial_{\mu} E_{b} \underline{\underline{d}}+\ldots
$$

where the dots denote connection terms. To evaluate the second term on the right-hand side we note that 


$$
\nabla_{\alpha} E_{b} \underline{\underline{d}}=E_{\alpha}{ }^{\mu} \partial_{\mu} E_{\beta} \underline{\underline{d}}+\ldots
$$

where the additional terms vanish at leading order in $\theta$. Since

$$
\begin{aligned}
\nabla_{\alpha} E_{b} \underline{c} & =K_{\alpha b} \gamma^{\prime} u_{c^{\prime}} \underline{c} \\
& =\left(\Gamma^{c^{\prime}}\right)_{\alpha \beta^{\prime}} \Lambda^{\beta^{\prime}} u_{c^{\prime}} \underline{c},
\end{aligned}
$$

this term can be evaluated straightforwardly. One finds

$$
\eta^{a b} E_{a}{ }^{\mu} \partial_{\mu} E_{b} \underline{d} \mid=-i \eta^{a b} \psi_{a}^{\alpha}\left(\Gamma^{c^{\prime}}\right)_{\alpha \beta^{\prime}} \lambda_{b}^{\beta^{\prime}} v_{c^{\prime}} \underline{d}
$$

where $\lambda=\Lambda \mid$. The connection terms in (4.39) involving the fermionic part of the connection $\Omega_{\mu}$ vanish at leading order and are therefore irrelevant. There are two bosonic connection terms, one for the (induced) worldvolume connection and one for the pulled-back target space connection. The latter is easily taken into account since it simply acts on underlined indices at $\theta=0$ via the Green-Schwarz pull-back, that is, the pull-back from the target superspace to the bosonic worldvolume. It is the former which needs careful evaluation. The term in question is $\eta^{a b}\left(-E_{a}{ }^{m} \Omega_{m, b}\right) \mid$. Now

$$
T_{a b}^{c}=\left(-E_{a}{ }^{M} \partial_{M} E_{b}{ }^{N} E_{N}{ }^{c}+\Omega_{a, b}{ }^{c}\right)-(a \leftrightarrow b)
$$

so that, at $\theta=0$, we have

$$
T_{a b}{ }^{c} \mid=\left(-e_{a}{ }^{m} \partial_{m} e_{b}{ }^{n} e_{n}{ }^{c}-\left(E_{a}{ }^{\mu} \partial_{\mu} E_{b}{ }^{n}\right) \mid e_{n}{ }^{c}+\omega_{a, b}{ }^{c}\right)-(a \leftrightarrow b) .
$$

This equation can be solved straightforwardly for $\omega$; the terms involving the vielbein alone give the Levi-Civita connection in an orthonormal basis associated with the GS metric, while the remaining terms give bilinear fermion contributions. The dimension one torsion is (4.13)

$$
T_{a b}^{c}=i \Lambda_{a}^{\alpha^{\prime}}\left(\Gamma^{c}\right)_{\alpha^{\prime} \beta^{\prime}} \Lambda_{b}{ }^{\beta^{\prime}}
$$

so that the left-hand side of (4.43) is easily evaluated. We also need to evaluate $E_{a}{ }^{m}$ at first order in $\theta$. Using $T_{\alpha \beta}{ }^{c}=-i\left(\Gamma^{c}\right)_{\alpha \beta}, T_{\alpha b}^{c}=0$ and a further choice of gauge one finds

$$
E_{\alpha}{ }^{m}=\frac{i}{2}\left(\Gamma^{a}\right)_{\alpha \beta} e_{a}^{m} \theta^{\nu} \delta_{\nu}^{\beta}+O\left(\theta^{2}\right)
$$

Using the relation between the supervielbein and its inverse we can then solve for $E_{\mu}{ }^{a}$ and then $E_{a}{ }^{m}$ at first order in $\theta$. The connection is therefore given by

$$
\omega_{a, b c}=\stackrel{o}{\omega}_{a, b c}+Y_{a, b c}
$$

where $\stackrel{o}{\omega}$ is the Levi-Civita connection and 


$$
Y_{a, b c}=k_{a, b c}+k_{b, a c}-k_{c, a b}
$$

with

$$
k_{a, b c}=-\frac{i}{2}\left(\lambda_{b}^{\beta^{\prime}}\left(\Gamma_{a}\right)_{\beta^{\prime} \gamma^{\prime}} \lambda_{c}^{\gamma^{\prime}}+\psi_{b}^{\beta}\left(\Gamma_{a}\right)_{\beta \gamma} \psi_{c}^{\gamma}\right)
$$

The terms with $\lambda$ vanish in the scalar equation of motion due to symmetry and the spin-half equation of motion. We therefore find

$$
\eta^{a b} \stackrel{o}{\nabla} a \mathcal{E}_{b} \underline{\underline{d}}=i \eta^{a b}\left(\psi_{a}^{\alpha}\left(\Gamma^{c^{\prime}}\right)_{\alpha \beta^{\prime}} \lambda_{b}^{\beta^{\prime}} v_{c^{\prime}} \underline{d}-\psi_{a}^{\alpha}\left(\Gamma_{b}\right)_{\alpha \beta} \psi^{c \beta} v_{c} \underline{d}+\ldots\right)
$$

where the omitted terms are due to the target space connection and four-form field strength. It is now straightforward to show that this can be rewritten in the desired form, namely

$$
\eta^{a b} \hat{\nabla}_{a} \mathcal{E}_{b} \underline{\underline{d}}=-\frac{1}{6} \epsilon^{a b c} \mathcal{E}_{c} \underline{\underline{C}} \mathcal{E}_{b} \underline{\underline{B}} \mathcal{E}_{a} \underline{\underline{A}} G_{\underline{A B C}} \underline{\underline{d}}
$$

where the covariant derivative is the Levi-Civita derivative with an additional term in the connection arising from the pull-back of the target space connection, and where

$$
\mathcal{E}_{a} \underline{A}=e_{a}^{m} \partial_{m} z^{\underline{M}} E_{\underline{M}}^{\underline{A}} \mid
$$

The bilinear fermion terms in (4.50) arise from the dimension zero component of $G_{4}$, namely, $G_{\underline{\alpha} \underline{\beta} \underline{c d}}=-i\left(\Gamma_{\underline{c d}}\right)_{\underline{\alpha \beta}}$.

\subsection{Kappa symmetry}

The basic embedding condition (2.5) is intimately related to $\kappa$-symmetry in the GS approach to branes $[10,11]$. Under an infinitesimal worldvolume diffeomorphism one has

$$
\left(\delta z^{\underline{M}}\right) E_{\underline{M}}^{\underline{A}}=v^{A} E_{A} \underline{A},
$$

where $v^{A}$ is the worldvolume vector field generating the diffeomorphism. For an odd diffeomorphism, with $v^{a}=0$, one finds, using the embedding condition,

$$
\delta z^{\underline{a}} \equiv\left(\delta z^{\underline{M}}\right) E_{\underline{M}}^{\underline{a}}=0,
$$

and

$$
\delta z^{\underline{\alpha}} \equiv\left(\delta z^{\underline{M}}\right) E_{\underline{M}}^{\underline{\alpha}}=v^{\alpha} E_{\alpha} \underline{\alpha} .
$$

This can be rewritten in the more familiar $\kappa$-symmetry form

$$
\delta z^{\underline{\alpha}}=\frac{1}{2} \kappa^{\underline{\beta}}(1+\Gamma)_{\underline{\beta}}^{\underline{\alpha}}
$$


where

$$
\kappa^{\underline{\alpha}}=v^{\alpha} E_{\alpha} \underline{\alpha}
$$

and where

$$
P_{\underline{\alpha}} \underline{\beta}=\frac{1}{2}(1+\Gamma)_{\underline{\alpha}} \underline{\beta}
$$

is the projection operator onto the odd tangent space of the worldvolume from the odd tangent space of the target ${ }^{2}$. It is given in terms of $E_{\alpha} \underline{\alpha}$ by

$$
P_{\underline{\alpha}}^{\underline{\beta}}=\left(E^{-1}\right)_{\underline{\alpha}}^{\gamma} E_{\gamma} \underline{\beta} \text {. }
$$

Thus we have

$$
\begin{aligned}
\delta z^{\underline{a}} & =0 \\
\delta z^{\underline{\alpha}} & =\frac{1}{2} \kappa^{\underline{\beta}}(1+\Gamma) \underline{\beta}^{\underline{\alpha}} .
\end{aligned}
$$

Equations (4.59), evaluated at $\theta=0$, are the standard $\kappa$-symmetry transformations of $z \underline{M}(x)$ in the GS formalism. The explicit form of the operator $\Gamma$, which must square to unity in order for $P$ to be a projector, and the explicit relation between the parameters for $\kappa$-symmetry and worldvolume supersymmetry depend on the choice of basis for the odd tangent space on the worldvolume, but whichever basis one chooses to work with, $\kappa$-symmetry has a precise definition in terms of worldvolume supergeometry. For our choice of basis it is easy to see, using Appendix A, that

$$
\begin{aligned}
\Gamma_{\underline{\alpha}}^{\underline{\beta}} & =-\frac{1}{6} \epsilon^{a b c}\left(\Gamma_{a b c}\right)_{\underline{\alpha}}^{\underline{\beta}} \\
& \rightarrow \delta_{\alpha}{ }^{\beta}\left(\begin{array}{cc}
\delta_{i}^{j} & 0 \\
0 & -\delta_{i^{\prime}} j^{\prime}
\end{array}\right)
\end{aligned}
$$

so that

$$
P_{\underline{\alpha}} \underline{\beta} \rightarrow \delta_{\alpha}^{\beta}\left(\begin{array}{cc}
\delta_{i}^{j} & 0 \\
0 & 0
\end{array}\right)
$$

as required.

\footnotetext{
${ }^{2} P$ and $\Gamma$ defined in the previous subsection are the $\theta=0$ components of corresponding objects in this subsection.
} 


\subsection{The action formula}

In this subsection we briefly review the action formula for arbitrary $p$-branes given in [22]. We shall then apply it to the M2-brane. For any brane we assume the existence of a closed Wess-Zumino form $W_{p+2}$ defined on the worldvolume $M$; it is given by

$$
W_{p+2}=d Z_{p+1}
$$

where $Z$ is a potential $(p+1)$-form. Since $W$ it is a $p+2$-form on a manifold which has even (i.e. bosonic) dimension $p+1$ it follows that it is exact. This is so because the de Rham cohomology of a supermanifold coincides with the de Rham cohomology of its body. Therefore we can always write it in the form

$$
W_{p+2}=d K_{p+1}
$$

for some globally defined $(p+1)$-form $K$ on $M$. Furthermore, since none of the target space fields or the worldvolume fields has negative dimension, at least at lowest order in derivatives, it follows that the only non-vanishing component of $K$ is the purely bosonic one. In components this means

$$
K_{\alpha A_{1} \cdots A_{p}}=0
$$

We now define the Green-Schwarz Lagrangian form $L_{p+1}$ to be

$$
L_{p+1}=K_{p+1}-Z_{p+1} .
$$

Under a worldvolume diffeomorphism generated by the vector field $v$ one has

$$
\delta L_{p+1}=\mathcal{L}_{v} L_{p+1}=d i_{v} L_{p+1}+i_{v} d L_{p+1}
$$

Since, by construction, $L_{p+1}$ is closed, the variation (4.66) reduces to

$$
\delta L_{p+1}=d i_{v} L_{p+1}
$$

Therefore the action integral

$$
S=\int_{M_{0}} L_{p+1}^{0}
$$

where $M_{0}$ is the body of $M$ and where

$$
L_{p+1}^{0}=d x^{m_{p+1}} \wedge d x^{m_{p}} \wedge \ldots d x^{m_{1}} L_{m_{1} \ldots m_{p+1}} \mid
$$

is invariant under $\kappa$-symmetry transformations and diffeomorphisms of $M_{0}$, since these transformations are identified with the leading components of the superdiffeomorphisms of $M$. The use of closed superspace $d$-forms to construct superinvariants in $d$ spacetime dimensions has been noted elsewhere in the literature [31, 32]. 
The Wess-Zumino form for the M2-brane is the pull-back of the supergravity four-form $G_{4}$. On the worldvolume of the brane there should therefore be a three-form $K_{3}$ such that

$$
W_{4}=f^{*} G_{4}=d K_{3}
$$

In index notation this reads

$$
4 \nabla_{[A} K_{B C D]}+6 T_{[A B}^{E} K_{|E| C D]}=\left(f^{*} G\right)_{A B C D} .
$$

Since there are no fields of negative dimension on the worldvolume (given the standard embedding condition), the only non-vanishing component of $K$ has purely even indices. By directly evaluating the dimension zero component of the (4.70) one finds that it is satisfied for

$$
K_{a b c}=\epsilon_{a b c} .
$$

It is then easy to verify that the entirety of (4.70) is satisfied [22]. In a coordinate basis one has

$$
K_{m n p} \mid=\epsilon_{m n p} \sqrt{-\operatorname{det} g},
$$

where $g$ is the GS metric. The GS Lagrangian is therefore recovered from the general formulae (4.65) and (4.68); it is [22]

$$
\mathcal{L}=\sqrt{-\operatorname{det} g}-\frac{1}{6} \epsilon^{m n p} \partial_{p} z^{\underline{P}} \partial_{n} z^{\underline{N}} \partial_{m} z^{\underline{M}} C_{\underline{M N P}},
$$

where $G_{4}=d C_{3}$ on $\underline{M}$, and where

$$
L^{0}=d x^{m} \wedge d x^{n} \wedge d x^{p} \epsilon_{m n p} \mathcal{L}
$$

\section{The induced supergeometry}

In this subsection it will be shown that the geometry induced on the worldvolume, discussed briefly in [13], is that of $d=3, N=8$ conformal supergravity. The induced multiplet is composite. This will be done by comparing the worldvolume geometry to the known superspace geometry of $d=3, N=8$ conformal supergravity [33]. In order to make the comparison it is convenient to redefine the worldvolume geometry such that the torsion constraints are in standard form. To simplify matters we shall take the target space to be flat (11|32)-dimensional superspace as the presence of non-trivial target space terms does not affect the structure of the worldvolume supergravity multiplet.

\subsection{The $d=3, N=8$ conformal supergravity multiplet}

We shall use tildes to distinguish worldvolume geometrical objects in standard form from the induced form partially listed in the preceding subsection. Since the dimension zero and one-half 
components of the induced torsion are already in standard form, the only amendments that need to be made are at dimension one. If we set

$$
\tilde{\Omega}_{a, b}^{c}=\Omega_{a, b}^{c}+M_{a, b}^{c}
$$

where

$$
M_{a, b c}=-\frac{i}{2}\left(\bar{\Lambda}_{a} \Gamma_{b} \Lambda_{c}-\bar{\Lambda}_{a} \Gamma_{c} \Lambda_{b}+\bar{\Lambda}_{b} \Gamma_{a} \Lambda_{c}\right)
$$

we find that

$$
\tilde{T}_{a b}^{c}=0
$$

and

$$
\tilde{T}_{a \beta}^{\gamma} \rightarrow \tilde{T}_{a \beta j}{ }^{\gamma k}=\delta_{j}^{k}\left(\gamma_{a}\right)_{\beta}^{\gamma} U+\left(\gamma^{b}\right)_{\beta}^{\gamma} V_{a b j}{ }^{k}
$$

where

$$
\begin{aligned}
U & =\frac{i}{8} \bar{\Lambda}^{a i^{\prime}} \Lambda_{a i^{\prime}} \\
V_{a b i j} & =-\frac{i}{8}\left(\sigma_{a^{\prime} b^{\prime}}\right)_{i j}\left(\sigma^{a^{\prime} b^{\prime}}\right)_{i^{\prime} j^{\prime}} \bar{\Lambda}_{a}^{i^{\prime}} \Lambda_{b}^{j^{\prime}} .
\end{aligned}
$$

The dimension one torsion is now in standard form (for a flat target space) so that the redefinition (5.1) is the only one that needs to be made in this case. The non-zero dimension one torsion for $d=3, N=8$ conformal supergravity has the same form as (5.4), except that $\delta_{j k} U$ is replace by $U_{j k}$ where the latter is a symmetric matrix of scalar fields. As we shall see in the next section, the particular form of $U$ that occurs here is a consequence of the choice of conformal gauge that we have made for the superembedding. However, neither of the leading components of $U$ or $V$ are fields in the conformal supergravity multiplet. To exhibit such a field it is necessary to compute the dimension one curvature $R_{\alpha i \beta j, k l}$. The field redefinition (5.1) does not change this component of the curvature so it can be calculated from the Gauss-Codazzi equations using our previous results. We find

$$
\begin{aligned}
\tilde{R}_{\alpha i \beta j, k l}= & i \epsilon_{\alpha \beta}\left(W_{i j k l}+2\left(\delta_{i k} \delta_{j l}-\delta_{j k} \delta_{i l}\right) U\right) \\
& +i\left(\gamma_{a}\right)_{\alpha \beta}\left(\delta_{i j} V_{k l}^{a}-4 \delta_{(i[k} V_{j) l]}^{a}\right)
\end{aligned}
$$

where

$$
W_{i j k l}=\frac{i}{64}\left(\sigma^{a^{\prime} b^{\prime}}\right)_{i j}\left(\sigma^{c^{\prime} d^{\prime}}\right)_{k l}\left(\sigma_{a^{\prime} b^{\prime} c^{\prime} d^{\prime}}\right)_{i^{\prime} j^{\prime}} \bar{\Lambda}^{a i^{\prime}} \Lambda_{a}^{j^{\prime}}
$$


This field is totally antisymmetric and anti-self-dual on the $S O(8)$ indices and is part of the conformal supergravity multiplet. One might consider it to be the super-Weyl tensor for $d=$ $3, N=8$ supergeometry.

There are additional, higher-dimensional fields in the supergravity multiplet but we shall not go into further details here. However, the calculations presented above show that the induced multiplet is indeed a conformal supergravity multiplet as claimed. The components of this multiplet are (in order of increasing dimension): $\left(e_{m}{ }^{a} ; \psi_{m}^{i} ; A_{m i j}, B_{i j k l} ; \rho_{i j k} ; C_{i j k l}\right)$, where each field is antisymmetric on its internal indices. As usual, $e$ is the graviton and $\psi$ represents the gravitini, while $A$ is the $S O(8)$ gauge field, $B$ and $C$ are scalar fields (anti-self-dual) and $\rho$ a set of spinor fields. The field $B$ is the leading component of the superfield $W$ so that it is the lowest-dimensional non-gauge field in the multiplet. Not counting gauge degrees of freedom one sees that this multiplet has $128+128$ degrees of freedom.

\subsection{Super-Weyl transformations}

We shall briefly discuss the effect of super-Weyl transformations on the worldvolume geometry since this establishes the fact that $U$ and $V$ do not belong to the conformal supergravity multiplet. We shall work with the standard geometry (marked by a tilde) and mark the transformed quantities by hats. The computations are most easily carried out using differential forms. The super-Weyl transformations are, for the basis forms,

$$
\begin{aligned}
& \tilde{E}^{a} \rightarrow \hat{E}^{a}=e^{-2 S} \tilde{E}^{a} \\
& \tilde{E}^{\alpha} \rightarrow \hat{E}^{\alpha}=e^{-S}\left(\tilde{E}^{\alpha}+\tilde{E}^{a} \Sigma_{a}{ }^{\alpha}\right)
\end{aligned}
$$

where

$$
\Sigma_{a}^{\alpha}=-2 i\left(\Gamma^{a}\right)^{\alpha \beta} \tilde{\nabla}_{\beta} S .
$$

Dually, for the basis vectors, we have

$$
\begin{aligned}
\hat{E}_{\alpha} & =e^{S} \tilde{E}_{\alpha} \\
\hat{E}_{a} & =e^{2 S}\left(\tilde{E}_{a}-\Sigma_{a}{ }^{\alpha} \tilde{E}_{\alpha}\right)
\end{aligned}
$$

For the connection one has

$$
\hat{\Omega}_{A}^{B}=\tilde{\Omega}_{A}^{B}+Y_{A}^{B}
$$

where, at dimension one-half,

$$
\begin{aligned}
Y_{\alpha i, b c} & =2\left(\gamma_{b c}\right)_{\alpha}{ }^{\beta} \Sigma_{\beta i} \\
Y_{\alpha i, j k} & =2\left(\delta_{i k} \Sigma_{\alpha j}-\delta_{i j} \Sigma_{\alpha k}\right)
\end{aligned}
$$


with $\Sigma_{\alpha i}:=\tilde{\nabla}_{\alpha i} S$. It follows that

$$
Y_{\alpha i, \beta j \gamma k}=\delta_{j k}\left(\epsilon_{\beta \alpha} \Sigma_{\gamma i}+\epsilon_{\gamma \alpha} \Sigma_{\beta i}\right)+2 \epsilon_{\beta \gamma}\left(\delta_{i k} \Sigma_{\alpha j}-\delta_{i j} \Sigma_{\alpha k}\right)
$$

At dimension one,

$$
\begin{aligned}
Y_{a, b c} & =2\left(\eta_{a b} \tilde{\nabla}_{c} S-\eta_{a c} \tilde{\nabla}_{b} S\right)+2 \bar{\Sigma}^{i} \Sigma_{i} \\
Y_{a, i j} & =L_{a i j}+i \bar{\Sigma}_{i} \gamma_{a} \Sigma_{j}
\end{aligned}
$$

where

$$
\tilde{\nabla}_{\alpha i} \tilde{\nabla}_{\beta j} S==i\left(\epsilon_{\alpha \beta} K_{i j}+\left(\gamma^{a}\right)_{\alpha \beta} L_{a i j}+\frac{i}{2} \delta_{i j}\left(\gamma^{a}\right)_{\alpha \beta} \tilde{\nabla}_{a} S\right)
$$

This equation should be read as defining the fields $K_{i j}$ and $L_{a i j}$ as components of the scalar superfield $S$. The former is symmetric while the latter is antisymmetric as $S O(8)$ tensors.

On substituting the above transformations into the expressions for the torsion and curvature with the standard constraints one finds that all the components of the torsion of dimension less or equal to one are unaffected except for the component $T_{a \beta}^{\gamma}$ for which one finds

$$
\hat{T}_{a \beta j}{ }^{\gamma k}=e^{2 S}\left(\tilde{T}_{a \beta j}{ }^{\gamma k}+2\left(\gamma_{a}\right)_{\beta}{ }^{\gamma} K_{j}^{\prime k}+2 \epsilon_{a}^{b c}\left(\gamma_{b}\right)_{\beta}{ }^{\gamma} L_{c j}^{\prime}{ }^{k}\right)
$$

where

$$
\begin{aligned}
K_{i j}^{\prime} & =K_{i j}-i \bar{\Sigma}_{i} \Sigma_{j}+\frac{i}{2} \delta_{i j} \bar{\Sigma}^{k} \Sigma_{k} \\
L_{a i j}^{\prime} & =L_{a i j}-i \bar{\Sigma}_{i} \gamma_{a} \Sigma_{j} .
\end{aligned}
$$

Thus, as expected, in a general conformal gauge, the component of the torsion tensor given in (5.16) is made up from a symmetric scalar and an antisymmetric vector. The result also shows that the leading components of $U$ and $V$ in (5.4) can be gauged to zero by an appropriate choice of the $\theta^{2}$ parameter in $S$. For the dimension one curvature we find

$$
\hat{R}_{\alpha i \beta j, k l}=e^{2 S}\left(\tilde{R}_{\alpha i \beta j, k l}+8 i \epsilon_{\alpha \beta} \delta_{[i[k} K_{j] l]}^{\prime}+2 i\left(\gamma_{a}\right)_{\alpha \beta}\left(\delta_{i j} L_{k l}^{\prime a}-4 \delta_{(i[k} L_{j) l]}^{\prime a}\right)\right) .
$$

This equation confirms that $W_{i j k l}$ can indeed be viewed as the super-Weyl tensor as it simply scales under super-Weyl transformations.

\section{The gauged M2-brane}

In this section we shall discuss gauged superembeddings for target superspaces which admit isometries. We shall focus on the case when there is just one $U(1)$ isometry, but the formalism 
can be straightforwardly generalised to the non-abelian isometries of group manifolds. ${ }^{3}$ In effect, this is a gauged sigma model in a superspace setting, but without a kinetic term for the gauge field associated with the isometry. As we shall see, this gauge field turns out to be the pull-back of the type IIA RR 1-form, but the worldvolume Born-Infeld gauge field appears in a natural manner in the action formula. While the $U(1)$ gauging of the supermembrane was discussed in [28] the role played by the $U(1)$ gauge field was apparently not fully appreciated there.

\subsection{Gauged M2-brane superembedding}

We consider the superembedding $f: M \rightarrow \underline{\hat{M}}$, from the worldvolume $M$ into the $D=11$ target superspace. We denote this by a hat as we shall use $\underline{M}$ for the $D=10$ IIA superspace, the two being related by the projection $\underline{p}: \underline{\hat{M}} \rightarrow \underline{M}$. The $U(1)$ isometry is generated by a vector field $\underline{\hat{K}}$ so that geometrical quantities will be annihilated by the Lie derivative along $\underline{\hat{K}}$. The gauge superembedding matrix is

$$
D f:=d f+C_{1} \otimes \underline{\hat{K}}
$$

where $C_{1}$ is the $U(1)$ gauge field on $M$. We denote the components of $D f$ with respect to standard bases on both superspaces by $\tilde{E}_{A} \underline{\hat{A}}$. We have

$$
\tilde{E}_{A} \underline{\hat{A}}=E_{A}^{M}\left(\partial_{M} z^{\underline{\hat{M}}}+C_{M} K \underline{\underline{\hat{M}}}\right) E_{\underline{\hat{M}}} \underline{\hat{A}} .
$$

It is convenient to follow the standard practice and work with the gauge-covariant pull-back defined by using $D f$ in place of $d f$. In other words, in a coordinate basis, $\partial_{M} z \underline{\hat{M}}$ is replaced by $\partial_{M} z^{\underline{\hat{M}}}+C_{M} K \underline{\hat{M}}$. Given a target-space form $\underline{\hat{\omega}}$ the gauge-covariant pull-back $\tilde{f} \underline{\hat{\omega}}$ can be written

$$
\tilde{f} \underline{\hat{\omega}}=f^{*} \underline{\hat{\omega}}+f^{*}\left(i_{\underline{\hat{K}}} \underline{\hat{\omega}}\right) \wedge C_{1}
$$

A useful identity is

$$
d \tilde{f} \underline{\hat{\omega}}=\tilde{f} d \underline{\hat{\hat{\omega}}}+G_{2} \wedge \tilde{f}_{\underline{\hat{K}}} \underline{\hat{\hat{\omega}}}+f^{*} \mathcal{L}_{\underline{\hat{K}} \underline{\hat{\omega}}} \wedge C_{1}
$$

where $G_{2}=d C_{1}$. The last term vanishes on invariant forms and so plays no rôle in the following considerations.

The torsion equation is modified slightly:

$$
2 \hat{\nabla}_{[A} \tilde{E}_{B]} \underline{\hat{C}}+T_{A B}{ }^{C} \tilde{E}_{C} \underline{\hat{C}}+G_{A B} K \underline{\hat{C}}=\tilde{E}_{B} \underline{\hat{\underline{B}}} \tilde{E}_{A} \underline{\underline{\hat{A}}} \hat{T}_{\underline{\hat{A} \hat{B}}}^{\hat{\underline{C}}},
$$

where $G_{2}=d C_{1}$. The covariant derivative $\hat{\nabla}$ in this formula involves a worldvolume connection acting on the worldvolume index of $\tilde{E}_{A} \underline{\underline{\hat{A}}}$ and the covariant pull-back of the target space connection acting on the target space index. It does not involve a separate term with the $U(1)$ gauge field because the embedding matrix is $U(1)$-invariant.

We choose the embedding condition to be the gauged version of the usual one, namely

\footnotetext{
${ }^{3}$ Non-abelian gauging in the Green-Schwarz model has been considered in [28].
} 


$$
\tilde{E}_{\alpha} \underline{\underline{a}}=0
$$

We can also parametrise the non-zero components of $\tilde{E}_{A} \underline{\underline{A}}$ in exactly the same way as the usual membrane with the aid of an element $\hat{u}$ of the eleven-dimensional Lorentz group. However, we are not allowed to set the field $h_{\alpha}{ }^{\beta^{\prime}}$ (introduced in (2.10)) to zero as the torsion equation has been modified. To make further progress we make a $10+1$ split of the even target space quantities. We put $z^{\underline{\hat{M}}}=\left(z^{\underline{M}}, y\right)$ and $E^{\underline{\hat{A}}}=\left(\hat{E}^{\underline{A}}, \hat{E}^{11}\right)^{4}$. The $A=\alpha, B=\beta, \underline{\hat{C}}=11$ component of the torsion equation reads

$$
T_{\alpha \beta}^{c} \tilde{E}_{c}^{11}+G_{\alpha \beta} \underline{\hat{K}}^{11}=\tilde{E}_{\alpha} \underline{\alpha}^{\underline{\underline{\beta}}} \underline{\hat{T}}_{\underline{\alpha \beta}}^{11}
$$

At this stage we note that we are free to impose a conventional constraint on $G_{\alpha \beta}$ by shifting the even part of $C$. The natural choice is to do this in such a way that

$$
T_{\alpha \beta}{ }^{c} \tilde{E}_{c}^{11}=0
$$

and this in turn implies that

$$
\tilde{E}_{A}^{11}=0
$$

This implies that, up to a gauge transformation, the worldvolume gauge field $C_{1}$ is the pull-back of the IIA target space RR 1-form $\underline{C}_{1}$,

$$
C_{1}=f_{o}^{*} \underline{C}_{1}
$$

where $f_{o}:=\underline{p} \circ f$ is the IIA superembedding. In components it is given by $z^{M} \rightarrow z \underline{M}(z)$. With the aid of (6.9) it is simple to show that

$$
\tilde{E}_{A} \underline{A}=E_{A} \underline{\underline{A}},
$$

where $E_{A} \underline{A}$ is the IIA embedding matrix. The $\underline{\hat{C}}=11$ component of the torsion equation now reads

$$
G_{A B} \underline{\hat{K}}^{11}=E_{B} \underline{\underline{B}} E_{A} \underline{\underline{A}}\left(\hat{T}_{\underline{A B}}^{11}-2 \hat{\Omega}_{[\underline{A B}]}^{11}\right)
$$

The connection component that appears in this equation comes from the $\hat{\nabla}$ covariant derivative in the torsion equation (6.5). As can be seen from (C.4) the expression in brackets on the righthand-side of (6.12) is the field strength tensor of the RR 1-form $\underline{C}$ multiplied by the scalar field $\Phi$. Since $\underline{\hat{K}}^{11}=\Phi$ we see that the field strength of the $U(1)$ gauge field is identified with the pull-back of the RR 2-form field strength as expected. The $\underline{C}$ component of the torsion equation now becomes the usual torsion equation of the D2-brane,

$$
2 \nabla_{[A} E_{B]}^{\underline{C}}+T_{A B}^{C} E_{C} \underline{\underline{C}}=E_{B} \underline{\underline{B}} E_{A} \underline{\underline{A}} T_{\underline{A B}}^{\underline{C}} .
$$

\footnotetext{
${ }^{4}$ The KK reduction of the supervielbein is described in Appendix C.
} 
In deriving this equation one has to use the fact that additional terms present in (6.5) cancel on using the Kaluza-Klein reduction of the $D=11$ superspace geometry given in appendix C.

\subsection{Geometrical structure, Wess-Zumino form and action}

The geometry of the preceding subsection can be clarified by the introduction of a $U(1)$ bundle $\hat{M}$ over $M$. We can define a gauged superembedding to be an equivariant map $\hat{f}: \hat{M} \rightarrow \underline{\hat{M}}$. Thus we have the following picture:

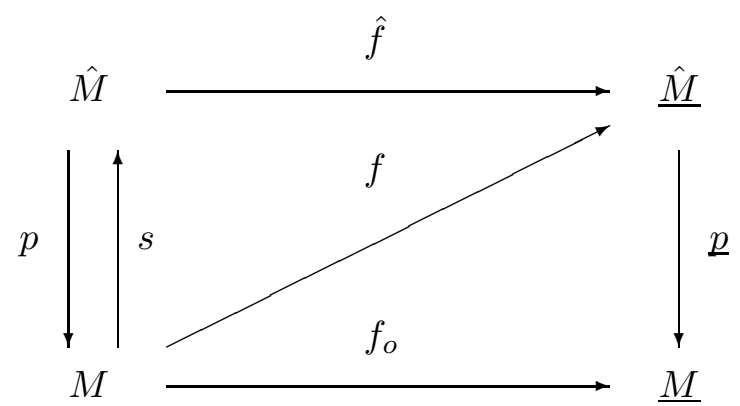

Here $p: \hat{M} \rightarrow M$ and $\underline{p}: \underline{\hat{M}} \rightarrow \underline{M}$ are the projections, $s: M \rightarrow \hat{M}$ is a section of $\hat{M}$, $f_{o}: M \rightarrow \underline{M}$ is the IIA superembedding, $\hat{f}: \hat{M} \rightarrow \underline{\hat{M}}$ is the lifted map and $f: M \rightarrow \underline{\hat{M}}$ is a gauged superembedding representative. The diagram is commutative so that the maps are related to each other in ways that can be read off straightforwardly. The equivariance of $\hat{f}$ means that

$$
\hat{f} \circ g=g \circ \hat{f}
$$

where $g$ denotes an element of $U(1)$ group acting on $\hat{M}$ on the left-hand side and $\underline{\hat{M}}$ on the right-hand side. From the diagram we have:

$$
\begin{aligned}
f & =\hat{f} \circ s \\
f_{o} & =\underline{p} \circ f=\underline{p} \circ \hat{f} \circ s .
\end{aligned}
$$

It should be emphasised that the above diagram and discussion is local. In general, we might have non-trivial bundles in which case neither $s$ nor $f$ is globally defined. In this case the equivariance of the map $\hat{f}$ still allows us to define $f_{o}$ uniquely from

$$
f_{o} \circ p=\underline{p} \circ \hat{f}
$$

Another way to think about the problem is that, given an embedding $f_{o}: M \rightarrow \underline{M}$ in IIA such that there is a $U(1)$ bundle $\underline{\hat{M}} \rightarrow \underline{M}$, then we can define $\hat{M} \rightarrow M$ as the pull-back of $\underline{\hat{M}}$. The maps $\hat{f}, s$ and $f$ can then be defined such that we obtain the above diagram. It is then natural to identify the connection form $\hat{\omega}$ of $\hat{M}$ with the pull-back under $\hat{f}$ of the connection form $\underline{\hat{\omega}}$ of $\underline{\hat{M}}$. 
The above discussion makes it clear that the gauged supermembrane in $D=11$ is simply a lifted version of the D2-brane in IIA. However, the formalism gives us a neat way to derive the D2 brane action directly starting from the generalised Wess-Zumino term without the need to dualise the additional transverse coordinate $y$ to a worldvolume Born-Infeld field.

As we shall see below, this construction requires the introduction of a field-strength 2 -form $\mathcal{F}_{2}$ on the worldvolume which obeys a modified Bianchi identity. This is the field strength of the gauge field on the brane. As the Wess-Zumino 4-form which we derive this way is exactly the same as the one obtained from studying the D2-brane directly in ten dimensions, it follows, provided we impose the usual embedding condition on $f_{o}$, that the brane action principle will lead us to the usual Green-Schwarz action complete with the Dirac-Born-Infeld kinetic term and the D-brane Wess-Zumino term.

Let $\underline{G}_{4}$ denote the closed 4 -form of $D=11$ supergeometry on $\underline{\hat{M}}$, and let $\hat{G}_{4}$ denote its pull-back to $\hat{M}$. We shall assume that $\underline{\hat{G}}_{4}$ is $U(1)$ invariant so that

$$
d \underline{\hat{G}}_{4}=\mathcal{L}_{\underline{\hat{K}}} \underline{\hat{G}}_{4}=0 .
$$

On the other hand $i_{\underline{\hat{K}}} \hat{\underline{G}}_{4}=\underline{H}_{3}$, the 3-form of type IIA. On $\hat{M}$, the pull-back $\hat{G}_{4}$ is again invariant and closed, and $i_{K} \hat{G}_{4}$ is closed, invariant and basic, that is, it satisfies (trivially) $\left.i_{K}\left(i_{K} \hat{G}_{4}\right)\right)=0$. It can therefore be expressed in terms of a 2 -form $\mathcal{F}_{2}$ on $\hat{M}$ which we can also assume to be invariant and basic. So we have

$$
i_{K} \hat{G}_{4}=-d \mathcal{F}_{2}
$$

where

$$
\mathcal{L}_{K} \mathcal{F}_{2}=i_{K} \mathcal{F}_{2}=0
$$

We would like to construct a Wess-Zumino 4 -form $W_{4}$ on $M$ in order to apply the brane action principle. The construction is essentially the same as that of [34] (see [35] for a review) but differs slightly in that we shall work on $\hat{M}$ rather than on $M$. In our approach a gauge-invariant Wess-Zumino form is equivalent to a closed, invariant, basic form $\hat{W}_{4}$ on $\hat{M}$ with $\hat{W}_{4}=p^{*} W_{4}$. Now $\hat{G}_{4}$ is not basic, but we can correct this by adding to it a term of the form $\omega \wedge i_{K} G_{4}$ where $\omega$ is the connection 1 -form on $\hat{M}$. This works because the connection form satisfies $i_{K} \omega=1$. The modified 4-form is no longer closed, but this in turn can be corrected by adding an extra term of the form $F_{\omega} \wedge \mathcal{F}_{2}$, where $F_{\omega}=d \omega=p^{*} G_{2}$ is the curvature 2-form. We thus arrive at a lifted Wess-Zumino 4-form

$$
\hat{W}_{4}=\hat{G}_{4}+\omega \wedge i_{K} G_{4}+F_{\omega} \wedge \mathcal{F}_{2}
$$

On $\underline{\hat{M}}$ we have

$$
\underline{\hat{G}}_{4}=\underline{G}_{4}+\underline{\hat{\omega}} \wedge \underline{H}_{3}
$$

where $\underline{\hat{\omega}}$ is the connection 1 -form of $\underline{\hat{M}}$. In standard coordinates this can be written $\underline{\hat{\omega}}=d \underline{y}+\underline{C}_{1}$ where $\underline{C}_{1}$ is the RR 1-form. Using this we find that $d \mathcal{F}_{2}=-H_{3}:=-f_{o}^{*} \underline{H}_{3}$ and so 


$$
W_{4}=G_{4}+G_{2} \mathcal{F}_{2}
$$

where $G_{2}=f_{o}^{*} \underline{G}_{2}=f_{o}^{*}\left(d \underline{C}_{1}\right)$. This is the standard Wess-Zumino form for the D2-brane. The corresponding potential is

$$
Z_{3}=f_{o}^{*} \underline{C}_{3}+f_{o}^{*} \underline{C}_{1} \wedge \mathcal{F}_{2}
$$

The usual Green-Schwarz action for the D2-brane can easily be reconstructed starting from $W_{4}$ with the aid of the brane action formula described in 4.4. This was done in detail in [22]; for the reader's convenience we outline the main steps.

We parametrise the dimension zero components of the embedding matrix in the form [15]

$$
\begin{aligned}
E_{\alpha} \underline{\underline{\alpha}} & =u_{\alpha} \underline{\underline{\alpha}}+h_{\alpha}^{\beta^{\prime}} u_{\beta^{\prime}} \underline{\alpha} \\
E_{a} \underline{a} & =u_{a} \underline{\underline{a}} .
\end{aligned}
$$

Here $u$ denotes part of a matrix of the group $\operatorname{Spin}(1,9)$, in the spinor or the vector representations according to the indices. An analysis of the dimension zero component of the torsion equation yields, on using the usual embedding condition $E_{\alpha} \underline{a}=0$,

$$
\begin{aligned}
h_{\alpha}{ }^{\beta^{\prime}} \rightarrow h_{\alpha i}{ }^{\beta^{\prime} j} & =\delta_{i}{ }^{j}\left(\gamma^{a b}\right)_{\alpha}{ }^{\beta^{\prime}} h_{a b} \\
T_{\alpha \beta}{ }^{c} & =-i\left(\Gamma^{d}\right)_{\alpha \beta} m_{d}{ }^{c},
\end{aligned}
$$

where

$$
m_{a}{ }^{b}=\delta_{a}{ }^{b}(1-4 y)+8\left(h^{2}\right)_{a}{ }^{b}, \quad y:=\frac{1}{2} \operatorname{tr} h^{2}
$$

and where $h^{2}$ denotes matrix multiplication. Using these results, one can show that the $\mathcal{F}$ identity, $d \mathcal{F}=-f_{o}^{*} H_{3}$, is satisfied provided that we choose all the components of $\mathcal{F}$ to vanish except for $\mathcal{F}_{a b}$ which is related to $h$ by

$$
m_{a}{ }^{c} \mathcal{F}_{c b}=4 h_{a b}
$$

This can be rearranged to give

$$
\mathcal{F}_{a b}=\frac{4 h_{a b}}{1+4 y}
$$

The Wess-Zumino four-form, $W_{4}=d\left(f^{*} C_{3}+f^{*} C_{1} \mathcal{F}\right)$, can be rewritten as $d K_{3}$. From the above constraints we find that all of the components of $K_{3}$ vanish except for $K_{a b c}$ which is given by

$$
K_{a b c}=\epsilon_{a b c} K
$$


with

$$
K=\frac{1-4 y}{1+4 y}
$$

$K_{3}$ is the same as for the M2-brane case except for the factor $K$ in $K_{a b c}$. As shown in [22] the above expression (6.31) for $K$ can be rewritten as

$$
K=\sqrt{\operatorname{det}\left(\delta_{m}^{n}+\mathcal{F}_{m}^{n}\right)} .
$$

The GS action is obtained by integrating the bosonic part of the closed 3-form $L_{3}=K_{3}-Z_{3}$ denoted by $L^{0}$. The Lagrangian is

$$
L^{0}=d x^{m} \wedge d x^{n} \wedge d x^{p} \epsilon_{m n p} \mathcal{L}
$$

with

$$
\mathcal{L}=\sqrt{-\operatorname{det}\left(g_{m n}+\mathcal{F}_{m n}\right)}-f^{*} C_{3}-f^{*} C_{1} \mathcal{F}
$$

This form of the Lagrangian is given in what we have called the KK frame in Appendix C. In the string frame the kinetic term in the action acquires a factor of $e^{-\phi}$.

\section{Massive branes}

Massive D-branes are D-branes whose worldvolume theories include a massive Chern-Simons term for the worldvolume gauge field. They were discussed in [29] where it was shown that kappa-symmetry requires the target space to be that of the Romans type IIA supergravity. Subsequently, it was shown how the massive D2-brane could be lifted to eleven dimensions, at least in components. In this section we shall discuss this lifting in the superembedding framework. In the next subsection we omit underlines and hats from eleven-dimensional quantities as the discussion does not involve any branes.

\subsection{Massive $D=11$ supergeometry}

In [27] it was shown how one rewrite massive IIA supergravity in eleven dimensions, although the resulting theory is not conventional $D=11$ supergravity since one has to introduce a Killing vector which cannot be eliminated. Here we apply a similar idea in the superspace context. Some partial results were obtained on this in [28].

The data in eleven dimensions is a vector field $K$ which generates the action of a circle group, a 3 -form gauge field $C_{3}$ and a set of 1 -forms $E^{A}$. All the fields we need to consider are invariant forms $\omega$, so that $\mathcal{L}_{K} \omega=0$. We can define a transformation on such forms in the following way:

$$
\delta \omega=m i_{K} \omega \wedge i_{K} \Lambda_{2}
$$


where the 2-form parameter $\Lambda_{2}$ is itself invariant. A covariant exterior derivative can be defined with the aid of the 3 -form gauge field $C_{3}$. If

$$
\delta C_{3}=d \Lambda_{2}+m i_{K} C_{3} \wedge i_{K} \Lambda_{2}
$$

then it is easy to check that

$$
D \omega:=d \omega+m i_{K} \omega \wedge i_{K} C_{3}
$$

transforms in the same way as $\omega$. The curvature 4 -form $G_{4}$ is defined by

$$
G_{4}=d C_{3}+\frac{m}{2} i_{K} C_{3} i_{K} C_{3}
$$

and is easily seen to transform covariantly.

The basis 1-forms $E^{A}$ transform in the usual way under local Lorentz transformations. Since $E^{A}$ is invariant under $K$, the Lorentz transformation parameter must be as well. $E^{A}$ is taken to transform under $\Lambda_{2}$ transformations as a 1-form so that the definition of the torsion needs to be modified as follows:

$$
T^{A}=D E^{A}=d E^{A}+E^{B} \Omega_{B}{ }^{A}+m i_{K} E^{A} i_{K} C_{3}
$$

This definition is similar to the one given in [27] for the bosonic part of the geometry, but with the difference that the extra term cannot be absorbed into the Lorentz connection as it does not have the right symmetry properties on the whole of superspace. The Bianchi identities which follow from this definition are

$$
D T^{A}=E^{B} R_{B}^{A}-m i_{K} E^{A} i_{K} G_{4}
$$

where the curvature is given by

$$
R_{A}{ }^{B}=d \Omega_{A}{ }^{B}+\Omega_{A}{ }^{C} \Omega_{C}{ }^{B}+m i_{K} \Omega_{A}{ }^{B} i_{K} C_{3} .
$$

Note that this curvature is Lorentz-valued and is covariant under both Lorentz and $\Lambda_{2}$ transformations. The Bianchi identity for the 4-form field strength is simply

$$
D G_{4}=0 \text {. }
$$

The constraints at dimension less than one on the curvatures for this geometry are the same as in the massless case, i.e. the only non-vanishing components of $T$ and $G$ are

$$
\begin{aligned}
T_{\alpha \beta}{ }^{c} & =-i\left(\Gamma^{c}\right)_{\alpha \beta} \\
G_{\alpha \beta c d} & =-i\left(\Gamma_{c d}\right)_{\alpha \beta}
\end{aligned}
$$

At dimension one we have the field-strength $G_{a b c d}$ which appears in the dimension one torsion $T_{a \beta}{ }^{\gamma}$ as before, but there are also mass-dependent corrections. The result is 


$$
T_{a \beta}^{\gamma}=\bar{T}_{a \beta}^{\gamma}+\frac{m}{12}\left(K^{2}\left(\Gamma^{c}\right)_{\alpha \beta}-6 m K_{a} K^{b}\left(\Gamma_{b}\right)_{\alpha \beta}\right)
$$

where $K^{2}=K^{a} K_{a}$ and $\bar{T}_{a \beta}{ }^{\gamma}$, given in (3.6), corresponds to the massless case. This can be obtained by examining the dimension one Bianchi identities (7.6). The mass-dependent terms originate from the second term on the right-hand side of (7.6).

\subsection{The massive gauged superembedding}

We shall now consider a gauged superembedding $f: M \rightarrow \underline{\hat{M}}$ where $\underline{\hat{M}}$ is the massive $D=11$ superspace described above. We define the gauged superembedding matrix as in the massless case. However, the presence of the mass term necessitates a further modification to the torsion identity which becomes

$$
2 \hat{\nabla}_{[A} \tilde{E}_{B]} \underline{\hat{C}}+T_{A B}^{C} \tilde{E}_{C} \underline{\hat{C}}+\mathcal{G}_{A B} K^{\underline{\hat{C}}}=\tilde{E}_{B} \underline{\hat{\underline{B}}} \tilde{E}_{A} \underline{\underline{\hat{A}}} \hat{T}_{\underline{\hat{A} \hat{B}}}^{\underline{\hat{C}}}
$$

where

$$
\mathcal{G}_{2}=G_{2}+m \tilde{f} i_{\underline{\hat{K}}} \underline{\hat{C}}_{3} .
$$

Note that this implies that

$$
d \mathcal{G}_{2}=m \tilde{f} i_{\underline{\hat{K}}} \underline{\hat{G}}_{4} .
$$

The only modification is in the change $G_{2} \rightarrow \mathcal{G}_{2}$ which comes from the covariant pull-back of the mass-dependent term in the torsion. Note that the covariant derivative in (7.12) contains only the induced Lorentz connection and the worldvolume connection. As usual we impose the embedding condition

$$
\tilde{E}_{\alpha} \underline{\hat{a}}=0
$$

Following a similar line of argument to the massless case, we make a $10+1$ split of the even coordinates and choose the bosonic part of the worldvolume gauge field $C_{1}$ such that the embedding trivialises in the eleventh direction, i.e. $\tilde{E}_{A}{ }^{11}=0$. This again implies that $C_{1}=f_{o}^{*} \underline{C}_{1}$ and that $\tilde{E}_{A} \underline{A}=E_{A} \underline{A}$. We now have (6.12) but with $G_{A B}$ replaced by $\mathcal{G}_{A B}$,

$$
\mathcal{G}_{A B} K^{11}=E_{B} \underline{\underline{B}} E_{A} \underline{\underline{A}}\left(\hat{\hat{T}}_{\underline{A B}}^{11}-2 \hat{\Omega}_{[\underline{A B}]}^{11}\right) .
$$

In this case it is the modified field strength of the $U(1)$ gauge field which is identified with the pull-back of the RR 2-form field strength. In other words

$$
\mathcal{G}_{2}=f_{o}^{*} \underline{G}_{2}
$$

Finally the $\underline{C}$ component of the torsion equation becomes the torsion equation of the massive D2-brane, 


$$
2 \nabla_{[A} E_{B]}^{\underline{C}}+T_{A B}^{C} E_{C} \underline{\underline{C}}=E_{B} \underline{\underline{B}} E_{A} \underline{\underline{A}} T_{\underline{A B}} \underline{C} .
$$

This is formally identical to the massless case, but the target superspace geometry is now that of massive IIA supergravity.

\subsection{The Wess-Zumino form}

We shall derive the massive D2-brane action starting from the covariant pull-back of $\hat{G}_{4}$. The idea is to add terms to $\tilde{f} \underline{G}_{4}$ in order to obtain a closed Wess-Zumino 4-form. Using (6.4) we find

$$
d \tilde{f} \underline{\hat{G}}_{4}=\mathcal{G}_{2} \wedge \tilde{f}\left(i_{\underline{\hat{K}}} \underline{\hat{G}}_{4}\right)
$$

Using (6.4) again we can easily show that $f\left(i_{\underline{\hat{K}}} \hat{\underline{G}}_{4}\right)$ is closed, so that we can write

$$
\tilde{f}\left(i_{\hat{K}} \underline{\hat{G}}_{4}\right)=-d \mathcal{F}_{2}
$$

where $\mathcal{F}_{2}$ is a worldvolume 2 -form which we identify with the field strength of the D2-brane gauge field. We then have

$$
d \tilde{f} \underline{\hat{G}}_{4}=-\mathcal{G}_{2} \wedge d \mathcal{F}_{2}=-d\left(\mathcal{G}_{2} \wedge \mathcal{F}_{2}\right)+d \mathcal{G}_{2} \wedge \mathcal{F}_{2}
$$

Equation (7.14) enables us to replace $d \mathcal{G}_{2}$ by $m \tilde{f}\left(i_{\underline{\underline{K}}} \underline{\hat{G}}_{4}\right)$ and this in turn is equal to $-m d \mathcal{F}_{2}$. The second term on the right of (7.21) is thus exact and we can therefore define the Wess-Zumino 4 -form $W_{4}$ to be

$$
W_{4}=\tilde{f} \underline{G}_{4}+\mathcal{G}_{2} \wedge \mathcal{F}_{2}+\frac{m}{2} \mathcal{F} \wedge \mathcal{F}
$$

Substituting the $10+1$ split into $W_{4}$, we see that the first term reduces to $f_{o}^{*} \underline{G}_{4}$,

$$
\tilde{f} \underline{\hat{G}}_{4}=\tilde{f}\left(\underline{G}_{4}+E^{11} \wedge\left(i_{\underline{\hat{K}}} \underline{\hat{G}}_{4}\right)\right)=f_{o}^{*} \underline{G}_{4}
$$

as $\tilde{f} E^{11}=0$. Using the fact that $\mathcal{G}_{2}=f_{o}^{*} \underline{G}_{2}$ we get

$$
W_{4}=f_{o}^{*} \underline{G}_{4}+\underline{G}_{2} \wedge \mathcal{F}_{2}+\frac{m}{2} \mathcal{F}_{2} \wedge \mathcal{F}_{2}
$$

This is the standard massive D2-brane Wess-Zumino form which can be written as $d Z_{3}$ where

$$
Z_{3}=f_{o}^{*} \underline{C}_{3}+f_{o}^{*} \underline{C}_{1} \wedge \mathcal{F}_{2}+\frac{m}{2} A d A
$$

The GS Lagrangian can be obtained by following the same steps as in the massless case. The only formal difference is the occurrence of the Chern-Simons term for the D-brane gauge field. Thus we find 


$$
\mathcal{L}=\sqrt{-\operatorname{det}\left(g_{m n}+\mathcal{F}_{m n}\right)}-f_{0}^{*} C_{3}-f_{0}^{*} C_{1} \mathcal{F}-\frac{m}{2} A d A
$$

in agreement with the result of reference [29].

\section{Conclusions}

In this paper we have provided the full details of the derivation of the M2-brane dynamics, including the equations of motion in a supergravity background in component form, in the framework of superembedding. We have used the brane action principle to derive the GreenSchwarz action for the supermembrane starting from a closed 4-form on the worldvolume, and we have shown how one can determine the worldvolume supervielbein and give expressions for the bosonic worldvolume dreibein and the worldvolume gravitino as composite fields. We have also studied the structure of the induced $d=3, N=8$ supergeometry and show that it coincides with that of off-shell $d=3, N=8$ conformal supergravity.

In section 6 we have introduced the notion of gauged superembedding and applied it to $U(1)$ gauging of the supermembrane in a suitable target space. We have shown that it leads directly to the D2-brane in Type IIA superspace without the need to dualise the additional scalar coming from the eleventh direction. We have done this at the level of the equations of motion via the natural gauged version of the embedding constraint; we have also shown how the D2-brane action can be derived using an appropriate modification of the construction of the Wess-Zumino term in gauged sigma models.

Finally, we have studied the massive D2-brane starting from $D=11$ and we have described the $D=11$ supergeometry corresponding to the lifted massive type IIA supergravity. While this is not truly eleven-dimensional, since it relies on the presence of a Killing vector, it has facilitated the construction of the brane theory via gauged superembedding. As in the massless case we have shown that the gauged superembedding formalism leads directly to the equations of motion of the D2-brane, and we have constructed the action which includes a Chern-Simons term for the Born-Infeld gauge field proportional to the mass.

\section{Acknowledgments}

We would like to than Per Sundell and Ulf Lindström for useful discussions. P.S.H. thanks PPARC for support under grant no PPA/G/O/2002/00475. The work of E.S. is supported in part by NSF grant PHY-0314712.

\section{A Conventions}

The following index conventions are used: plain (underlined) indices refer to worldvolume and target space quantities respectively and primed indices refer to normal spaces; indices from the beginning of the alphabet refer to preferred bases, indices from the middle of the alphabet to coordinate bases; Latin (Greek) indices are used for even (odd) indices, while capital indices 
run over the whole space. Thus a worldvolume preferred basis index could be $A=(a, \alpha)$ while a target space coordinate index could be $\underline{M}=(\underline{m}, \underline{m})$. Tensor quantities with indices are not underlined, but if the indices are omitted or if a target space tensor is projected on some of its indices we underline the tensor.

In sections 6 and 7 we use plain indices for the worldvolume, underlined indices for tendimensional IIA superspace and underlined hatted indices for eleven-dimensional superspace.

For the spinor indices a two step notation is used. Initially the target space index $\underline{\alpha}$, running from 1 to 32 is split in two, $\underline{\alpha} \rightarrow\left(\alpha, \alpha^{\prime}\right)$, where both the worldvolume index $\alpha$ and the normal index $\alpha^{\prime}$ run from 1 to 16 . For some purposes this is adequate but sometimes one wishes to recognise explicitly that these indices transform under the spin group in $d=p+1$ dimensions times an internal symmetry group. For the membrane we set

$$
\begin{aligned}
\psi^{\alpha} & \rightarrow \psi^{\alpha i} \\
\psi^{\alpha^{\prime}} & \rightarrow \psi^{\alpha i^{\prime}}
\end{aligned}
$$

where the spinor index on the right takes on two values while $i$ and $i^{\prime}$ both run from 1 to 8 .

We use space-favoured metrics throughout, $\eta_{a b}=(-1,+1, \ldots,+1)$, and the spacetime $\epsilon$-tensors all have $\epsilon^{0123 \cdots}=+1$.

We use the following representation of the $D=11 \Gamma$-matrices:

$$
\begin{aligned}
\Gamma^{a} & =\gamma^{a} \otimes \gamma_{9} \\
\Gamma^{a^{\prime}} & =1 \otimes \gamma^{a^{\prime}}
\end{aligned}
$$

where the $d=3 \gamma$-matrices are $2 \times 2$ and the $d^{\prime}=8 \gamma$-matrices are $16 \times 16$. The charge conjugation matrix is

$$
C=\epsilon \otimes \gamma_{9}
$$

In indices

$$
\begin{gathered}
\left(\Gamma^{a}\right)_{\underline{\alpha}} \underline{\beta}=\left(\gamma^{a}\right)_{\alpha}^{\beta}\left(\begin{array}{cc}
\delta_{i}^{j} & 0 \\
0 & -\delta_{i^{\prime}} j^{\prime}
\end{array}\right) \\
\left(\Gamma^{a^{\prime}}\right)_{\underline{\alpha}}^{\underline{\beta}}=\delta_{\alpha}{ }^{\beta}\left(\begin{array}{cc}
0 & \left(\sigma^{a^{\prime}}\right)_{i} j^{j^{\prime}} \\
\left(\tilde{\sigma}^{a^{\prime}}\right)_{i^{\prime}} j & 0
\end{array}\right)
\end{gathered}
$$

and

$$
C_{\underline{\alpha \beta}}=\epsilon_{\alpha \beta}\left(\begin{array}{cc}
\delta_{i j} & 0 \\
0 & -\delta_{i^{\prime} j^{\prime}}
\end{array}\right)
$$


where the $8 \times 8 \sigma$-matrices are related to the eight-dimensional $\gamma$-matrices by

$$
\gamma^{a^{\prime}}=\left(\begin{array}{cc}
0 & \left(\sigma^{a^{\prime}}\right)_{i j^{\prime}} \\
\left(\tilde{\sigma}^{\alpha^{\prime}}\right)_{i^{\prime} j} & 0
\end{array}\right)
$$

Eleven-dimensional spinor indices are raised or lowered according to the rule

$$
\psi^{\underline{\alpha}}=C \underline{\alpha \underline{\beta}} \psi_{\underline{\beta}} \leftrightarrow \psi_{\underline{\alpha}}=\psi^{\underline{\beta}} C_{\underline{\beta}} \underline{\alpha}
$$

where $C$ with upper indices is the same matrix as $C$ with lower indices. Three-dimensional spinor indices are similarly raised or lowered using $\epsilon$. Eight-dimensional indices, whether spinor or vector, are raised and lowered with the standard Euclidean metric. The $\Gamma$-matrices with lowered indices are

$$
\begin{aligned}
\left(\Gamma^{a}\right)_{\underline{\alpha \beta}} & =\left(\gamma^{a}\right)_{\alpha \beta}\left(\begin{array}{cc}
\delta_{i j} & 0 \\
0 & \delta_{i^{\prime} j^{\prime}}
\end{array}\right) \\
\left(\Gamma^{a^{\prime}}\right)_{\underline{\alpha \beta}} & =\epsilon_{\alpha \beta}\left(\begin{array}{cc}
0 & -\left(\sigma^{a^{\prime}}\right)_{i j^{\prime}} \\
\left(\tilde{\sigma}^{a^{\prime}}\right)_{i^{\prime} j} & 0
\end{array}\right)
\end{aligned}
$$

It is straightforward to decompose any of the eleven-dimensional $\Gamma$-matrices with multi-vector indices in this way. We give the two-index $\Gamma$-matrices as they are used most in the text:

$$
\begin{aligned}
\left(\Gamma^{a b}\right)_{\underline{\alpha \beta}} & =\left(\gamma^{a b}\right)_{\alpha \beta}\left(\begin{array}{cc}
\delta_{i j} & 0 \\
0 & \delta_{i^{\prime} j^{\prime}}
\end{array}\right) \\
\left(\Gamma^{a b^{\prime}}\right)_{\underline{\alpha \beta}} & =-\left(\gamma^{a}\right)_{\alpha \beta}\left(\begin{array}{cc}
0 & -\left(\sigma^{b^{\prime}}\right)_{i j^{\prime}} \\
\left(\tilde{\sigma}^{b^{\prime}}\right)_{i^{\prime} j} & 0
\end{array}\right) \\
\left(\Gamma^{a^{\prime} b^{\prime}}\right)_{\underline{\alpha \beta}} & =\epsilon_{\alpha \beta}\left(\begin{array}{cc}
\left(\sigma^{a^{\prime} b^{\prime}}\right)_{i j^{\prime}} & 0 \\
0 & -\left(\tilde{\sigma}^{a^{\prime} b^{\prime}}\right)_{i^{\prime} j}
\end{array}\right)
\end{aligned}
$$

The three-dimensional $\gamma$-matrices are real, symmetric with lowered indices, and satisfy

$$
\gamma^{a} \gamma^{b}=\eta^{a b}+\gamma^{a b}
$$

where

$$
\gamma^{a b}=\epsilon^{a b c} \gamma_{c} \leftrightarrow \gamma_{a}=-\frac{1}{2} \epsilon_{a b c} \gamma^{b c}
$$

For the eight-dimensional $\sigma$-matrices one may take

$$
\begin{aligned}
& \sigma^{a^{\prime}}=\left(1, i \tau_{r}\right) \\
& \tilde{\sigma}^{a^{\prime}}=\left(1,-i \tau_{r}\right)
\end{aligned}
$$


where $r=1, \ldots 7$, and where the $\tau_{r}$ are seven-dimensional Dirac matrices which are purely imaginary and antisymmetric. The matrices $\sigma^{a^{\prime} b^{\prime} c^{\prime} d^{\prime}}$ and $\tilde{\sigma}^{a^{\prime} b^{\prime} c^{\prime} d^{\prime}}$ are symmetric, the former being self-dual, the latter anti-self-dual, $\sigma^{a^{\prime} b^{\prime}}$ and $\tilde{\sigma}^{a^{\prime} b^{\prime}}$ are antisymmetric.

\section{B Riemannian embeddings}

We briefly review some elements of the theory of Riemannian embeddings. In this section $M$ is an $n$-dimensional manifold embedded in an $\underline{n}$-dimensional Riemannian manifold $(\underline{M}, \underline{g})$. We recall that the main features of an embedding are: the determination of the induced tensors on $M$ and the splitting of the tangent bundle $\underline{T}$ (restricted to $M$ ) into tangent and normal subbundles $T$ and $T^{\prime}$; the Gauss-Weingarten equations which define the induced connections on $T$ and $T^{\prime}$ and which introduce the second fundamental form; the torsion equations which relate the target space torsion tensor to the induced worldvolume torsion, and the Gauss-Codazzi equations which express the curvature tensors of $T$ and $T^{\prime}$ in terms of the target space curvature and the second fundamental form.

In the case of Riemannian embeddings the metric on $\underline{M}$ induces a metric on $M$ in the obvious way, and we can furthermore demand that $T^{\prime}$ and $T$ are orthogonal. This gives rise to a fibre metric on $T^{\prime}$. We let $\left(e_{a}\right)$ be a basis of vectors on $M,\left(e_{a}\right)$ a basis of vectors on $\underline{M}$ and $\left(e_{a^{\prime}}\right)$ a basis for the normal bundle $\left(\operatorname{dim} T^{\prime}=n^{\prime}\right)$. We have

$$
e_{a}=e_{a} \underline{a} e_{\underline{a}}
$$

and

$$
e_{a^{\prime}}=e_{a^{\prime}} \underline{a} e_{\underline{a}}
$$

We shall assume that the basis $\left(e_{\underline{a}}\right)$ is orthonormal. We can define a natural induced Riemannian structure on $M$ by taking the basis $\left(e_{a}\right)$ to be orthonormal,

$$
\underline{g}\left(e_{a}, e_{b}\right)=\delta_{a b}
$$

We take the normal space to be orthogonal to the tangent space to $M$, and the basis $\left(e_{a^{\prime}}\right)$ to be orthonormal with respect to $\underline{g}$ as well. Thus

$$
\begin{aligned}
\underline{g}\left(e_{a}, e_{b^{\prime}}\right) & =0 \\
\underline{g}\left(e_{a^{\prime}}, e_{b^{\prime}}\right) & =\delta_{a^{\prime} b^{\prime}}
\end{aligned}
$$

Clearly the above induces an $O(n)$ structure on $M$ and an $O\left(n^{\prime}\right)$ structure on $T^{\prime}$.

We can interpret the embedding matrix $e_{a}^{\underline{\underline{a}}}$ as a section of $T^{*} \otimes \underline{T}$ and the normal matrix $e_{a^{\prime}} \underline{a}$ as a section of $T^{*} \otimes \underline{T}$. We define covariant derivatives of these tensors, both denoted by $\nabla$ since it should be clear from the context which one is meant, by

$$
\begin{aligned}
& \nabla_{a} e_{b} \underline{\underline{c}}=e_{a} e_{b} \underline{\underline{c}}-\omega_{a, b}{ }^{c} e_{c^{\underline{c}}}+\omega_{a, \underline{b}} \underline{\underline{c}} u_{b} \underline{\underline{b}} \\
& \nabla_{a} e_{b^{\prime}} \underline{\underline{c}}=e_{a} e_{b}^{\prime \underline{c}}-\omega_{a, b^{\prime}} c^{c^{\prime}} e_{c^{\prime}}^{\underline{c}}+\omega_{a, \underline{b}} e_{b^{\prime}} \underline{\underline{c}}
\end{aligned}
$$


where $\omega_{a, \underline{b}} \underline{\underline{c}}$ is the target space connection pulled back onto the world surface, $\omega_{a, b}{ }^{c}$ is a connection for $T$ and $\omega_{a, b^{\prime}} c^{\prime}$ is a connection for $T^{\prime}$. These connections are not as yet specified in terms of the target space connection. The Gauss-Weingarten equations, which do just this, can be written

$$
\begin{aligned}
\nabla_{a} e_{b} \underline{\underline{c}} & =K_{a, b}{ }^{c^{\prime}} e_{c^{\prime}} \underline{c} \\
\nabla_{a} e_{b^{\prime}} \underline{c} & =K_{a, b^{\prime}} e_{c^{\underline{c}}}
\end{aligned}
$$

where $K_{a, b}{ }^{c^{\prime}}$ is the second fundamental form of the surface and $K_{a, b^{\prime}}{ }^{c}$ is related to it via equation (B.9) below. It is easy to show that equations (B.6) are indeed the Gauss-Weingarten equations. To see this, we note that they imply

$$
\nabla_{a}\left(Y^{b} e_{b}^{\underline{c}}\right)=\left(\nabla_{a} Y^{b}\right) e_{b}^{\underline{c}}+Y^{b} K_{a, b} c^{\prime} e_{c^{\prime}}^{\underline{c}}
$$

and

$$
\nabla_{a}\left(Y^{b^{\prime}} e_{b^{\prime}} \underline{c}\right)=\left(\nabla_{a} Y^{b^{\prime}}\right) e_{b}^{\prime} \underline{c}+Y^{b^{\prime}} K_{a, b^{\prime}} e_{c^{\underline{c}}}^{\underline{c}}
$$

where $Y$ and $Y^{\prime}$ are tangential and normal vector fields respectively. On the left-hand side of both equations the $T$-indices are contracted out so that the covariant derivative is simply the covariant derivative of the target space $\underline{M}$. On the right-hand side of (B.7) the first term is tangential and defines the induced connection for $T$ while the second term is normal and defines the second fundamental form. Similarly, on the right-hand side of (B.8) the first term is normal and defines the induced connection on $T^{\prime}$ while the second term is tangential. It is easy to show that the connections defined by the Gauss-Weingarten equations are metric and that

$$
K_{a, b c^{\prime}}=-K_{a, c^{\prime} b}
$$

One way of doing this is to define

$$
K_{a}:=\left(\nabla_{a} e\right) e^{-1}
$$

where $e$ is the $\underline{n} \times \underline{n}$ matrix formed from $e_{a} \underline{a}$ and $e_{a^{\prime}} \underline{a}$. The equations

$$
K_{a, b}^{c}=0
$$

and

$$
K_{a, b^{\prime}} c^{\prime}=0
$$

are equivalent to the Gauss-Weingarten equations above. Since $\left(e_{a}\right)$ and $\left(e_{a^{\prime}}\right)$ are orthonormal bases it follows that $e$ is an element of $O(\underline{n})$ and hence that $K$ is an $\mathfrak{o}(\underline{n})$-valued one-form. From this one derives (B.6) immediately, while it is straightforward to show that the induced connections for $T$ and $T^{\prime}$ are metric since they are $\mathfrak{o}(n)$ and $\mathfrak{o}\left(n^{\prime}\right)$ valued respectively.

The torsion 2 -form of $\underline{M}$ is given by

$$
T^{\underline{a}}=d e^{\underline{a}}+e^{\underline{b}} \wedge \omega_{\underline{b}}^{\underline{a}}
$$


where $(e \underline{a})$ is the basis of forms dual to $\left(e_{\underline{a}}\right)$ and $\omega_{\underline{b}} \underline{\underline{c}}:=e^{\underline{a}} \underline{\omega}_{\underline{a}, \underline{b}} \underline{\underline{c}}$. When pulled back onto $M$ the right-hand side becomes

$$
d\left(e^{a} e_{a}^{\underline{a}}\right)+e^{a} e_{a} \underline{\underline{b}} \underline{\omega}_{\underline{\underline{b}}}^{\underline{a}}=D\left(e^{a} e_{a}^{\underline{a}}\right)
$$

where $D$ is the covariant exterior derivative for the $\underline{T}$ index. Since the non-underlined indices are contracted one has

$$
D\left(e^{a} e_{a}^{\underline{a}}\right)=T^{a} e_{a}^{\underline{a}}+e^{b} \wedge e^{a} \nabla_{b} e_{a}^{\underline{a}}
$$

from which one arrives at

$$
\nabla_{a} e_{b}^{\underline{c}}-\nabla_{b} e_{a}^{\underline{c}}+T_{a b}^{c} e_{c}^{\underline{c}}=e_{a}^{\underline{a}} e_{b}^{\underline{b}} T_{\underline{a b}}^{\underline{c}} .
$$

Multiplying this equation by $e_{\underline{c}}^{c}$ one has

$$
T_{a b}^{c}=e_{a}^{\underline{a}} e_{b} \underline{b} T_{\underline{a b}} \underline{c} e_{\underline{c}}^{c}
$$

using the induced connection condition. Thus the torsion of $M$ is related to the torsion of $\underline{M}$ in a simple fashion and clearly vanishes if the latter does. If we multiply (B.16) by $e_{\underline{c}}{ }^{\prime}$ we find

$$
K_{a b} c^{c^{\prime}}-K_{b a}{ }^{c^{\prime}}=e_{a} \underline{\underline{a}} e_{b}^{\underline{b}} T_{\underline{a} \underline{b}} \underline{\underline{c}} e_{\underline{c}}^{c^{\prime}}
$$

so that the second fundamental form is symmetric if the target space torsion vanishes.

¿From the definition of $K$ it is easy to derive the following relation by differentiation

$$
\nabla_{a} K_{b}-\nabla_{b} K_{a}+T_{a b}{ }^{c} K_{c}-\left[K_{a}, K_{b}\right]=\left(R_{a b} e\right) e^{-1}
$$

where the curvature operator acts on both indices of $e$ (with a minus sign for the lower indices). Taking the $\mathfrak{o}(n)$ and $\mathfrak{o}\left(n^{\prime}\right)$ parts of this equation we get the equations of Gauss and Codazzi, which are, for the case of induced connections,

$$
\begin{aligned}
& R_{a b, c}{ }^{d}=\underline{R}_{a b, c}{ }^{d}+\left(K_{a, c}{ }^{e^{\prime}} K_{b, e^{\prime}}{ }^{d}-(a \leftrightarrow b)\right) \\
& R_{a b, c^{\prime}} d^{\prime}=\underline{R}_{a b, c^{\prime}} d^{d^{\prime}}+\left(K_{a, c^{\prime}}{ }^{e} K_{b, e}{ }^{d^{\prime}}-(a \leftrightarrow b)\right)
\end{aligned}
$$

where

$$
\underline{R}_{a b, c}{ }^{d}=e_{a} \underline{a}_{b} e^{\underline{b}} e_{c}^{\underline{c}} R_{\underline{a b}, \underline{c}} \underline{d}^{\left(e^{-1}\right)_{\underline{d}}^{d}}
$$

and

$$
\underline{R}_{a b, c^{\prime}} d^{\prime}=e_{a}^{\underline{a}} e_{b} \underline{\underline{b}} e_{c^{\prime}} \underline{\underline{c}} R_{\underline{a b}, \underline{\underline{d}}} \underline{\underline{d}}\left(e^{-1}\right)_{\underline{d}}^{d^{\prime}} .
$$




\section{Kaluza-Klein reduction of $D=11$ superspace}

The reduction of supergravity formulated in $D=11$ superspace to ten dimensions was outlined in [5], but the full details were not given there. Here we provide them. It is convenient to do this in two steps: first, we make a simple reduction to what we shall refer to as the KK frame, and then we rescale the supervielbein to go to the string frame. The formulae specifying the KK reduction are

$$
\begin{aligned}
& \underline{E}^{A}=E^{A}+E^{11} \chi^{A} \\
& \underline{E}^{11}=\Phi E^{11}
\end{aligned}
$$

where eleven-dimensional quantities are underlined and $A$ denotes a $D=10$ type IIA superindex. The object $\chi^{A}$ vanishes for $A=a$, while for $A=\alpha$ it denotes the spinor field of IIA supergravity. The scalar field $\Phi$ is related to the dilaton in a way which will be made precise below and the one-form $E^{11}$ is defined by

$$
E^{11}=d y+C_{1}
$$

where $C_{1}$ is the RR one-form gauge field. For the connection we put

$$
\begin{aligned}
\underline{\Omega}_{a}{ }^{b}= & \Omega_{a}{ }^{b}+E^{11} \Omega_{y a}{ }^{b} \\
\underline{\Omega}_{a}{ }^{11}= & \Omega_{a}{ }^{11}+E^{11} \Omega_{y a}{ }^{11} \\
\underline{\Omega}_{\alpha}{ }^{\beta}= & \Omega_{\alpha}{ }^{\beta}+\frac{1}{2}\left(\Gamma^{a} \Gamma_{11}\right)_{\alpha}{ }^{\beta} \Omega_{a}{ }^{11} \\
& +E^{11}\left(\Omega_{y \alpha}{ }^{\beta}+\frac{1}{2}\left(\Gamma^{a} \Gamma_{11}\right)_{\alpha}{ }^{\beta} \Omega_{y a}{ }^{11}\right) .
\end{aligned}
$$

These formulae define a permissible choice of the IIA connection. The additional components of the $D=11$ connection can be read off from the torsion. On using the above expressions in the formula for the torsion one finds

$$
\begin{aligned}
\underline{T}_{A B}{ }^{c} & =T_{A B}{ }^{c} \\
\underline{T}_{A B}^{\gamma} & =T_{A B}{ }^{\gamma}+G_{A B} \chi^{\gamma}+\Omega_{[A, c}{ }^{11}\left(\Gamma^{c} \Gamma_{11}\right)_{B]}{ }^{\gamma} \\
\underline{T}_{A B}{ }^{11} & =\Phi G_{A B}+\Omega_{[A, B]}{ }^{11}
\end{aligned}
$$

together with three further equations involving the additional connection components. In the above equation quantities which are not defined for particular values of the super indices are taken to be zero, e.g. $\Omega_{A, \beta}{ }^{11}$, while $G=d C_{1}$ is the RR field strength 2 -form. After some algebraic manipulation one finds 


$$
\begin{aligned}
T_{\alpha \beta}^{c} & =-i\left(\Gamma^{c}\right)_{\alpha \beta} \\
T_{\alpha b}{ }^{c} & =T_{a b}^{c}=0 \\
T_{\alpha \beta}{ }^{\gamma} & \left.=i \Phi^{-1}\left(\Gamma_{11}\right)_{\alpha \beta} \chi^{\gamma}-i \Phi^{-1}\left(\Gamma^{c} \chi\right)_{(\alpha}\left(\Gamma_{c} \Gamma_{11}\right)_{\beta}\right)^{\gamma} \\
T_{a \beta}{ }^{\gamma} & =\underline{T}_{a \beta}^{\gamma}-G_{a \beta} \chi^{\gamma}+\frac{1}{4} G_{a b}\left(\Gamma^{b} \Gamma_{11}\right)_{\beta} \gamma .
\end{aligned}
$$

The components of $G_{A B}$ are also determined by the reduction procedure. They are

$$
\begin{aligned}
G_{\alpha \beta} & =-i \Phi^{-1}\left(\Gamma_{11}\right)_{\alpha \beta} \\
G_{\alpha b} & =-i \Phi^{-2}\left(\Gamma_{b} \chi\right)_{\alpha}
\end{aligned}
$$

and $G_{a b}$. We also find that

$$
\chi_{\alpha}=\nabla_{\alpha} \Phi
$$

The additional components of the eleven-dimensional connection are given by

$$
\begin{aligned}
\Omega_{\alpha, b 11} & =i \Phi^{-1}\left(\Gamma_{b} \chi\right)_{\alpha} \\
\Omega_{a, b 11} & =-\frac{1}{2} \Phi G_{a b} \\
\Omega_{y, b 11} & =\nabla_{a} \Phi \\
\Omega_{y, a b} & =\frac{1}{2} \Phi^{2} G_{a b} .
\end{aligned}
$$

The components of the IIA 3 -and 4-form field strengths can be read off using the formulae

$$
\begin{aligned}
G_{A B C D} & =\underline{G}_{A B C D} \\
H_{A B C} & =\Phi \underline{G}_{A B C 11}-\chi^{\delta} \underline{G}_{\delta A B C} .
\end{aligned}
$$

One finds that the non-vanishing components are

$$
\begin{aligned}
G_{\alpha \beta c d} & =-i\left(\Gamma_{c d}\right)_{\alpha \beta} \\
G_{a b c d} & =\underline{G}_{a b c d} \\
H_{\alpha \beta c} & =-i \Phi\left(\Gamma_{c} \Gamma_{11}\right)_{\alpha \beta} \\
H_{\alpha b c} & =-i\left(\Gamma_{b c} \chi\right)_{\alpha} \\
H_{a b c} & =\Phi \underline{G}_{a b c 11} .
\end{aligned}
$$


The string frame can be reached by the rescalings

$$
E^{a} \mapsto e^{\frac{\phi}{3}} E^{a} \quad E^{\alpha} \mapsto e^{\frac{\phi}{6}} E^{\alpha}
$$

provided that we set

$$
\Phi=e^{\frac{2 \phi}{3}}
$$

where $\phi$ is the dilaton. It is also necessary to redefine the bosonic part of the connection if one wishes to maintain the standard constraint $T_{a b}{ }^{c}=0$, but we shall not bother to do this here. The new components of the torsion up to dimension one-half in the string frame are

$$
\begin{aligned}
T_{\alpha \beta}{ }^{c} & =-i\left(\Gamma^{c}\right)_{\alpha \beta} \\
T_{\alpha b}{ }^{c} & =\delta_{b}{ }^{c} \lambda_{\alpha} \\
T_{\alpha \beta}{ }^{\gamma} & =-2\left(\Gamma_{11}\right)_{\alpha \beta}\left(\Gamma_{11} \lambda\right)^{\gamma}+2\left(\Gamma^{c} \Gamma_{11} \lambda\right)_{(\alpha}\left(\Gamma_{c} \Gamma_{11}\right)_{\beta)}{ }^{\gamma}+\delta_{(\alpha}{ }^{\gamma} \lambda_{\beta)}
\end{aligned}
$$

where we have introduced

$$
\lambda_{\alpha}:=\frac{1}{3} \nabla_{\alpha} \phi
$$

For the non-zero components of the form field strengths up to dimension one-half we have

$$
\begin{aligned}
G_{\alpha \beta} & =-i e^{-\phi}\left(\Gamma_{11}\right)_{\alpha \beta} \\
G_{\alpha b} & =2 e^{-\phi}\left(\Gamma_{b} \Gamma_{11} \lambda\right)_{\alpha} \\
G_{\alpha \beta c d} & =-i e^{-\phi}\left(\Gamma_{c d}\right)_{\alpha \beta} \\
H_{\alpha \beta c} & =-i\left(\Gamma_{c} \Gamma_{11}\right)_{\alpha \beta} \\
H_{\alpha b c} & =2\left(\Gamma_{b c} \Gamma_{11} \lambda\right)_{\alpha} .
\end{aligned}
$$

These results seem to be compatible with the expressions given for the superspace tensors of type IIA supergravity given in references $[36,37]$ but we were not able to find a redefinition which would take them into the form given in [29].

\section{References}

[1] E. Bergshoeff, E. Sezgin and P. K. Townsend, "Supermembranes and Eleven-Dimensional Supergravity," Phys. Lett. B 189 (1987) 75.

[2] M. J. Duff and K. S. Stelle, "Multi-Membrane Solutions of D = 11 Supergravity," Phys. Lett. B 253 (1991) 113. 
[3] B. de Wit, J. Hoppe and H. Nicolai, "On the Quantum Mechanics of Supermembranes," Nucl. Phys. B 305 (1988) 545.

[4] T. Banks, W. Fischler, S. H. Shenker and L. Susskind, "M theory as a Matrix Model: A Conjecture," Phys. Rev. D 55 (1997) 5112 [arXiv:hep-th/9610043].

[5] M. J. Duff, P. S. Howe, T. Inami and K. S. Stelle, "Superstrings in D = 10 From Supermembranes In D = 11," Phys. Lett. B 191 (1987) 70.

[6] M. J. Duff and J. X. Lu, "Type II p-branes: The Brane Scan Revisited," Nucl. Phys. B 390 (1993) 276 [arXiv:hep-th/9207060].

[7] P. K. Townsend, "D-branes from M-branes," Phys. Lett. B 373 (1996) 68 [arXiv:hepth/9512062].

[8] M. B. Green and J. H. Schwarz, "Covariant Description of Superstrings," Phys. Lett. B 136 (1984) 367.

[9] W. Siegel, "Hidden Local Supersymmetry in the Supersymmetric Particle Action," Phys. Lett. B 128 (1983) 397.

[10] D. P. Sorokin, V. I. Tkach and D. V. Volkov, "Superparticles, Twistors and Siegel Symmetry," Mod. Phys. Lett. A 4 (1989) 901.

[11] D. P. Sorokin, V. I. Tkach, D. V. Volkov and A. A. Zheltukhin, "From the Superparticle Siegel Symmetry to the Spinning Particle Proper Time Phys. Lett. B 216 (1989) 302.

[12] D. P. Sorokin, "Superbranes and Superembeddings," Phys. Rept. 329, 1 (2000) [arXiv:hepth/9906142].

[13] I. A. Bandos, D. P. Sorokin, M. Tonin, P. Pasti and D. V. Volkov, "Superstrings and Supermembranes in the Doubly Supersymmetric Geometrical Approach," Nucl. Phys. B 446 (1995) 79 [arXiv:hep-th/9501113].

[14] P. S. Howe and E. Sezgin, "Superbranes," Phys. Lett. B 390 (1997) 133 [arXiv:hepth/9607227].

[15] P. S. Howe and E. Sezgin, "D = 11, p = 5," Phys. Lett. B 394 (1997) 62 [arXiv:hepth/9611008].

[16] P. S. Howe, E. Sezgin and P. C. West, "Covariant Field Equations of the M-Theory Fivebrane," Phys. Lett. B 399 (1997) 49 [arXiv:hep-th/9702008].

[17] P. Pasti, D. P. Sorokin and M. Tonin, "Covariant Action for a D = 11 Five-brane with the Chiral Field," Phys. Lett. B 398 (1997) 41 [arXiv:hep-th/9701037].

[18] M. Aganagic, J. Park, C. Popescu and J. H. Schwarz, "Worldvolume Action of the M-theory Five-brane," Nucl. Phys. B 496 (1997) 191 [arXiv:hep-th/9701166].

[19] P. S. Howe, E. Sezgin and P. C. West, "Aspects of Superembeddings," arXiv:hepth/9705093.

[20] C. S. Chu, P. S. Howe and E. Sezgin, "Strings and D-branes with Boundaries," Phys. Lett. B 428 (1998) 59 [arXiv:hep-th/9801202]. 
[21] I. A. Bandos, D. P. Sorokin and D. Volkov, "On the Generalized Action Principle for Superstrings and Supermembranes," Phys. Lett. B 352 (1995) 269 [arXiv:hep-th/9502141].

[22] P. S. Howe, O. Raetzel and E. Sezgin, "On Brane Actions and Superembeddings," JHEP 9808 (1998) 011 [arXiv:hep-th/9804051].

[23] P. S. Howe, S. F. Kerstan, U. Lindstrom and D. Tsimpis, "The Deformed M2-brane," JHEP 0309 (2003) 013 [arXiv:hep-th/0307072].

[24] J. M. Drummond and S. F. Kerstan, "Kappa-symmetric Deformations of M5-brane dynamics," arXiv:hep-th/0412149.

[25] J. M. Drummond and S. F. Kerstan, "Kappa-symmetric Derivative Corrections to D-brane Dynamics," JHEP 0410 (2004) 006 [arXiv:hep-th/0407145].

[26] T. Ortin, "A Note on the D-2-brane of the Massive Type IIA Theory and Gauged Sigma Models," Phys. Lett. B 415 (1997) 39 [arXiv:hep-th/9707113].

[27] E. Bergshoeff, Y. Lozano and T. Ortin, "Massive Branes," Nucl. Phys. B 518 (1998) 363 [arXiv:hep-th/9712115].

[28] H. Nishino and S. Rajpoot, "Supermembrane with U(1)-gauging," Eur. Phys. J. C 39 (2005) 389 [arXiv:hep-th/0309100].

[29] E. Bergshoeff, P. M. Cowdall and P. K. Townsend, "Massive IIA Supergravity from the Topologically Massive D2-brane," Phys. Lett. B 410 (1997) 13 [arXiv:hep-th/9706094].

[30] P. S. Howe, "Weyl Superspace," Phys. Lett. B 415 (1997) 149 [arXiv:hep-th/9707184].

[31] R. D'Auria, P. Fre, P. K. Townsend and P. van Nieuwenhuizen, "Invariance Of Actions, Rheonomy and the New Minimal N=1 Supergravity in the Group Manifold Approach," Annals Phys. 155 (1984) 423.

[32] S. J. J. Gates, M. T. Grisaru, M. E. Knutt-Wehlau and W. Siegel, "Component Actions from Curved Superspace: Normal Coordinates and Ectoplasm," Phys. Lett. B 421 (1998) 203 [arXiv:hep-th/9711151].

[33] P. S. Howe, J. M. Izquierdo, G. Papadopoulos and P. K. Townsend, "New Supergravities with Central Charges and Killing Spinors in (2+1)-dimensions," Nucl. Phys. B 467 (1996) 183 [arXiv:hep-th/9505032].

[34] C. M. Hull and B. Spence, "The Geometry of the Gauged Sigma Model with Wess-Zumino Term," Nucl. Phys. B 353 (1991) 379.

[35] R. Percacci and E. Sezgin, "Properties of Gauged Sigma Models," arXiv:hep-th/9810183.

[36] J. L. Carr, S. J. J. Gates and R. N. Oerter, "D = 10, N=2a Supergravity in Superspace," Phys. Lett. B 189 (1987) 68.

[37] M. Cederwall, A. von Gussich, B. E. W. Nilsson, P. Sundell and A. Westerberg, "The Dirichlet Super-p-branes in Ten-dimensional Type IIA and IIB Supergravity," Nucl. Phys. B 490 (1997) 179 [arXiv:hep-th/9611159]. 